\title{
The size-frequency distribution of $H>13$ NEOs and ARM target candidates detected by Pan-STARRS1
}

\author{
Eva Schunová - Lilly ${ }^{1}$ (lilly@ifa.hawaii.edu) \\ Robert Jedicke $^{1}$, Peter Vereš ${ }^{2,3}$, Larry Denneau ${ }^{1}$ Richard J. Wainscoat $^{1}$
}

Received

accepted

43 Pages, 11 Figures, 0 Tables

\footnotetext{
${ }^{1}$ Institute for Astronomy University of Hawaii, 2680 Woodlawn Dr, Honolulu, HI, 96822, USA

${ }^{2}$ Department of Astronomy, Physics of the Earth and Meteorology, Comenius University, Mlynská dolina, Bratislava, 942 48, Slovakia

${ }^{3}$ NASA Jet Propulsion Laboratory, 4800 Oak Grove Dr, Pasadena, CA 91109, USA
} 


\begin{abstract}
We determine the absolute magnitude $(\mathrm{H})$ distribution (or size-frequency distribution, $\mathrm{SFD} ; N(H) \propto 10^{\alpha H}$ where $\alpha$ is the slope of the distribution) for near-Earth objects (NEO) with $13<H<30$ and Asteroid Retrieval Mission (ARM) targets with $27<H<31$ that were detected by the $1^{\text {st }}$ telescope of the Panoramic Survey Telescope and Rapid Response System (Pan-STARRS1; e.g. Kaiser et al. 2002; Kaiser 2004; Hodapp et al. 2004). The NEO and ARM target detection efficiencies were calculated using the Greenstreet et al. (2012) NEO orbit distribution. The debiased Pan-STARRS1 NEO absolute magnitude distribution is more complex than a single slope power law - it shows two transitions - at $\mathrm{H} \sim 16$ from steep to shallow slope, and in the $21<H<23$ interval from a shallow to steep slope, which is consistent with other recent works (e.g. Mainzer et al. 2011c; Brown et al. 2013; Harris and D'Abramo 2015). We fit $\alpha=0.48 \pm 0.02$ for NEOs with $13<H<16, \alpha=0.33 \pm 0.01$ for NEOs with $16<H<22$, and $\alpha=0.62 \pm 0.03$ for the smaller objects with $H>22$. There is also another change in slope from steep to shallow around $\mathrm{H}=27$. The three ARM target candidates detected by Pan-STARRS1 in one year of surveying have a corrected SFD with slope $\alpha=0.40_{-0.45}^{+0.33}$.

We also show that the window for follow up observations of small $(\mathrm{H} \gtrsim 22)$ NEOs with the NASA IRTF telescope and Arecibo and Goldstone radars are extremely short - on order of days, and procedures for fast response must be implemented in order to measure physical characteristics of small Earth-approaching objects. CFHT's MegaCam and Pan-STARRS1 have longer observing windows and are capable of following-up more NEOs due to their deeper limiting magnitudes and wider fields of view.
\end{abstract}


Key Words: Near-Earth Objects, Asteroids, Asteroids, dynamics

\section{Introduction}

The near-Earth objects (NEOs), asteroids or comets orbiting the Sun with perihelion distance $q<1.3 \mathrm{AU}$, are both potentially threatening and beneficial - the orbits of many NEOs bring them close to Earth so they may eventually impact our planet, but this also makes them the most easily accessible of all the objects in the Solar system for commercial (e.g. asteroid mining) and scientific purposes. In this paper we calculate Pan-STARRS1's (e.g. Kaiser 2004; Hodapp et al. 2004) NEO and Asteroid Retrieval Mission (ARM; Abell et al. 2015) target detection efficiency and use it to determine an unbiased number distribution of both types of objects as a function of their absolute magnitude.

Attention has recently shifted from large NEOs with diameters $>1 \mathrm{~km}$ to smaller NEOs ( $<100 \mathrm{~m}$ diameter) as they now harbor the residual Earth impact risk and because they are particularly convenient space mission targets. The potential of even small NEOs to cause localized damage must not be underestimated - the Chelyabinsk airburst of an $\sim 17 \mathrm{~m}$ diameter object over Russia on 15 February 2013 injured about 1500 people (e.g. Brown et al. 2013; Borovička et al. 2013) and the Tunguska event in 1908 flattened trees over $\sim 2000 \mathrm{~km}^{2}$ (e.g. Andreev et al. 1993).

Relatively little is known about small NEOs as they are usually faint due to their small size and thus difficult to discover unless they make a close Earth approach. While more than $90 \%$ of NEOs with $D>1 \mathrm{~km}$ are known, only $\sim 25 \%$ of those with $D>100 \mathrm{~m}$ have been discovered and the population completeness drops down to $10^{-5}$ for $\mathrm{D} \sim 10 \mathrm{~m}$ objects (Harris and D'Abramo 2015; Mainzer et al. 2011a).

Brown et al. (2002) derived the small NEO SFD from $8.5 \mathrm{yr}$ of satellite detections of 
airbursts (explosions produced by small asteroids with $D<10 \mathrm{~m}$ entering the atmosphere). Their results are consistent with the SFD extrapolated from lunar cratering records (Ivanov 2006; Ito and Malhotra 2010). Recently, Brown et al. (2013) suggest that a steeper slope in the SFD in this size range might be evidence for non-equilibrium in the population of small Earth impactors. This would imply that the number of impactors with $\mathrm{D} \sim 10-50 \mathrm{~m}$ may be an order of magnitude higher than estimates based on other techniques. On the other hand, Harris and D'Abramo (2015) argue this claim should be dismissed because their results based on survey simulations agree with Brown et al. (2013) to a factor of two for this size and their SFD estimates are likely accurate within the factor of three over the whole NEO size range.

The knowledge of composition and internal structure of small NEOs is very fragmentary due to the lack of data. Smaller NEOs were thought to be monolithic rocks based on the observational data suggesting they can rotate faster than the stability limit of a gravitationally bound rubble pile(e.g. Pravec and Harris 2000). However, Scheeres et al. (2010) suggest that some may still be rubble piles because cohesive Van der Waals forces between small grains are capable of holding them together. These fast-rotating small NEOs may be the terminal state of $\mathrm{NEO}$ evolution driven by the $\mathrm{YORP}^{1}$ effect (e.g. Bottke et al. 2002b). This theory is supported by Mommert et al. (2014a,b) who obtained infrared measurements and high precision astrometry of two small NEAs, 2009 BD and 2011 MD. The estimated bulk densities of these objects ranges from about 600 to $1800 \mathrm{~kg} \mathrm{~m}^{-3}$ suggesting that both asteroids are indeed rubble-piles.

Finally, Brown et al. (2016) finds there is more than an order of magnitude spread in the strength of small NEOs impacting Earth and suggests they may be best considered

\footnotetext{
${ }^{1}$ The Yarkovsky-O'Keefe-Radziebskii-Paddack (YORP) effect can alter the spin rate of irregularly shaped objects under the influence of asymmetric thermal re-radiation
} 
as a continuum which extends from very strong monolithic objects to weakly bound sand castles.

Small NEOs are ideal objects for a detailed geological study as they can be relatively easily retrieved as samples. Particularly interesting are objects on low inclination, low eccentricity orbits with semi-major axis similar to Earth. The cratering record measurements on the Moon suggest the existence of a population of low speed projectiles (Ito and Malhotra 2010), which might contain suitable targets for e.g. NASA's ARM mission.

NASA has now decided to retrieve a large boulder from an asteroid and deliver it to lunar orbit (Abell et al. 2015) but an alternative mission design was to identify, rendezvous with, and redirect an entire small NEO of $\sim 10 \mathrm{~m}$ diameter to lunar orbit. The target population for the latter ARM mission scenario is discussed in $§ 2.2$ and referred to as 'the ARM target population' because these objects remain scientifically interesting and could be candidates for future in situ resource utilization missions. The ARM targets need to be accessible with low $\Delta v$ and must be neither too small nor too large in order to be effective resources.

A specific problem regarding the small NEO population is the short time available for follow up observations and characterization after the discovery. Due to their typically high apparent motion follow up observation is necessary within hours otherwise the orbital uncertainty grows too rapidly and the object is inevitably lost. A short observing window also makes the physical characterization of small NEOs challenging, requiring rapid response in scheduling the observation and possible cooperation between observatories (Mommert et al. 2016; Thirouin et al. 2016). §3.4 describes this issue in greater detail and concludes with our measurement of the observing windows of NEOs and ARM targets as a function of the absolute magnitude for multiple optical, IR telescopes and radar facilities. 


\section{Method}

To determine the actual NEO population's absolute magnitude distribution for $13<H<30$ and the ARM target population with $27<H<31$ we developed a synthetic data processing pipeline to measure the performance of a simulated Pan-STARRS1 survey (denoted as PS1* through the rest of the paper). First, we generated a synthetic population according to the Greenstreet et al. (2012) NEO model. We generated independent populations of NEOs and ARM targets in 1.0 and 0.5 magnitude wide $H$ bins respectively and assigned objects within each bin an absolute magnitude according to the Brown et al. (2002) NEO SFD. The number of objects generated in each bin was sufficient to ensure good statistics in the synthetic discovered population. To reduce the processing requirements NEOs and ARM targets that met a size-dependent Minimum Orbit Intersection Distance (MOID) requirement with Earth were injected into the Pan-STARRS1 Moving Object Processing System (MOPS; Denneau et al. 2013) to simulate the survey. The synthetic detections identified by MOPS were then post-processed to account for tracklet identification efficiency and trailing losses to mimic the real Pan-STARRS1 survey. The remaining synthetic detections were then used to calculate the PS1* NEO and ARM target identification efficiency. These steps are described further in the following sections.

\subsection{NEO Model}

The synthetic NEO orbit distribution was generated according to the Greenstreet et al. (2012) model that corrects several deficiencies of the long-standing Bottke et al. (2002a) NEO model including 1) having higher resolution in semi-major axis $(a)$, eccentricity $(e)$, and inclination $(i), 2)$ using higher statistics integrations, with 3) a finer time resolution, and 4) incorporating retrograde NEOs. The first point is particularly important to the detection of NEOs accessible to human space missions because the Bottke et al. (2002a) 
NEO model includes relatively few bins covering the range of ARM target candidate orbits.

The Greenstreet et al. (2012) NEO model uses the same weighting as the Bottke et al. (2002a) model for the different main belt NEO sources but their finer time and orbital element resolution results in some important differences between the orbit distributions (fig. 1). A Kolmogorov-Smirnov (KS) comparison of both models with the $(a, e, i)$ distributions of known NEOs with $H<18$ (a nearly complete population) reveals that neither model formally agrees with the observed NEO distribution at the $95 \%$ confidence level. Despite the formal disagreement, the Bottke et al. (2002a) model's semi-major axis distribution agrees better with the observed NEOs while the Greenstreet et al. (2012) model is a better match in both eccentricity and inclination. Furthermore, our calculation of the detection efficiency as a function of absolute magnitude is relatively insensitive to the details of the underlying 'hidden' orbit distribution, and the uncertainties on the debiased SFD will be limited by the detection statistics, not the underlying model's systematic errors.

It is more difficult to compare the orbit element distributions at smaller sizes because of observational selection effects but Mainzer et al. (2011b) suggest that the models under-predict the number of objects on low-inclination orbits. Ito and Malhotra (2010) also suggest the existence of a previously undetected population of slow, low-speed impacting objects responsible for the rayed crater distribution on the Moon (both works set no restrictions on the size of the objects). Furhtermore, current NEO models do not include the contribution of lunar ejecta. Large impacts on the Moon could have provided a population of small low $\Delta v$ NEOs akin to $1991 \mathrm{VG}$ (Tancredi 1998), which would be good targets for space mission (e.g. ARM).

This work focuses on NEOs with $H>13(D \lesssim 8.5 \mathrm{~km})^{2}$ observed by Pan-STARRS1 during calendar years 2014 and 2015 when operations were stable and the system was

\footnotetext{
${ }^{2}$ unless otherwise stated we use a mean geometric albedo of $\rho=0.15$ from
} 
devoted nearly $100 \%$ to NEO surveying. We limit our study to $H<30$ ( $D \gtrsim 5 \mathrm{~m}$ ) because 1) we know that the detection efficiency is exceedingly small for objects in this size range (as of August 2016 there are only 26 known NEOs with $H>30$ ), 2) this size overlaps the range of the largest bolides (Brown et al. 2016), and 3) it overlaps with our ARM target sample (see section $§ 2.2$ ).

We generated synthetic NEOs in each of 17 one magnitude-wide bins in the $13 \leq H<30$ range and assigned each an absolute magnitude within the bin with a probability $\propto 10^{0.54 H}$ (Brown et al. 2002). The shape of the $H$ distribution within a bin is not particularly important because we will calculate the detection efficiency on a bin-by-bin basis, but our $H$ bins are relatively wide so the Brown et al. (2013) SFD was employed to increase the fidelity of the efficiency calculation in the $H>25$ bins where the efficiency changes most quickly. We generate enough synthetics in each bin that the simulated survey (\$2.5) would detect $>100$ objects per bin (before implementing system losses) so the uncertainty on the corrected number of PS1* objects is not dominated by the statistical uncertainty in our efficiency determination. This required the generation of 300 million synthetic objects in the bins corresponding to the smallest objects with the lowest detection efficiency.

\subsection{ARM target model}

The ARM target population is dynamically restricted by five criteria ${ }^{3}$ :

i) $0.7 \mathrm{AU}<q<1.05 \mathrm{AU}$

ii) $0.95 \mathrm{AU}<Q<1.45 \mathrm{AU}$

iii) $2.99233<T_{E}<3.01$

http://sbn.psi.edu/pds/resource/albedo.html

3 Chodas et al., JPL, (personal communication) 
iv) $e>-1.40591 \frac{a}{\mathrm{AU}}+1.33562$

v) $e>+0.89132 \frac{a}{\mathrm{AU}}-0.93588$

where $q=a(1-e)$ and $Q=a(1+e)$ are an object's perihelion and aphelion distances respectively, and $T_{E}$ is its Tisserand parameter with respect to Earth,

$$
T_{E}=\frac{\mathrm{AU}}{a}+2 \cos i \sqrt{\frac{a}{\mathrm{AU}}\left(1-e^{2}\right)} .
$$

Their dynamical limits naturally restrict the objects' accessibility for a spacecraft mission as measured by the candidate's $v_{\infty}$ and the required mission $\Delta v$. We use Shoemaker and Helin (1978) to estimate the $\Delta v$ required to rendezvous with the object in two maneuvers beginning from low-Earth orbit, and estimate the object's excess speed above Earth's escape speed ${ }^{3}$ with $v_{\infty} \sim 29.76 \sqrt{3-T_{E}} \mathrm{~km} \mathrm{sec}^{-1}$. As of August 2016 there were only 41 known objects meeting all the requirements described below.

The ARM targets' Earth-like orbits and small sizes make them distinct from the generic NEO population described above (\$2.1). Only about $0.0030 \%$ of NEOs in the Greenstreet et al. (2012) model pass the ARM target selection cuts, yielding a population of objects on extremely Earth-like orbits (fig. 2) with $a \sim 1 \mathrm{AU}$, small eccentricities $(e \lesssim 0.15)$ and low inclinations $\left(i \lesssim 6^{\circ}\right)$. Due to the selection criteria $73 \%$ of the ARM targets have orbits with $\Delta v<5 \mathrm{~km} \mathrm{sec}^{-1}$ and all of them have $v_{\infty}<3.6 \mathrm{~km} \mathrm{sec}^{-1}$, both conditions being required for a fuel- and cost-efficient spacecraft-asteroid rendezvous and return mission. For comparison only $0.2 \%$ of the known NEO population has $\Delta v<5 \mathrm{~km} \mathrm{sec}^{-1}$ and only $0.3 \%$ of them fulfill the condition that $v_{\infty}<3.6 \mathrm{~km} \mathrm{sec}^{-1}$.

The Greenstreet et al. (2012) NEO model has only $8 a$-bins, 8 -bins and $3 i$-bins spanning the range of the ARM targets's orbital elements so that the generation of random synthetic objects according to the model created a strong and unphysical signature of the bin edges in the resulting $(a, e, i)$ distributions (fig. 2). This would have an unrealistic effect on the predicted ARM target detection rates due to e.g. clustering of objects along 
the bin edges, and artificially enhancing the number of objects with $i \sim 0^{\circ}$. We overcame this difficulty by fitting the Greenstreet et al. (2012) NEO model to empirically selected functions in the $192(=8 \times 8 \times 3)(a, e, i)$ bins corresponding to the ARM targets. First, we verified that the distributions in each parameter are roughly independent and then the semi-major axis distribution was fit to a quartic function of the form

$f_{a}(a)=-893.337+3563.68(a / \mathrm{AU})^{1}-5141.61(a / \mathrm{AU})^{2}+3204.28(a / \mathrm{AU})^{3}-724.582(a / \mathrm{AU})^{4}$,

the eccentricity to a gaussian of the form

$$
f_{e}(e)=43.9115 \exp ^{-\frac{1}{2}\left(\frac{e-0.184212}{0.067361}\right)^{2}}
$$

and the inclination to a line forced to be zero at $i=0^{\circ}$

$$
f_{i}(i)=13.7509(i / \mathrm{deg})^{1}
$$

The normalization of each of the functions is immaterial when generating random $a, e$ and $i$. We then generated random sets of $(a, e, i)$ and applied the ARM target dynamical cuts to select objects from our smoothed sample. This 'smoothed' model allowed us to rapidly generate synthetic ARM targets and eliminated the bin edge effects (fig. 2).

The ARM targets's absolute magnitudes are restricted to the range $27 \leq H<31$ corresponding to diameters $2<D<30 \mathrm{~m}$ depending on their albedo. We generated objects in eight 0.5-magnitude wide bins in this size range in the same manner as for the NEOs

described above using the Brown et al. (2002) NEO SFD. We used 0.5 magnitude wide bins because we expect the efficiency to quickly drop somewhere in this range.

\subsection{MOID selection}

The fraction of all NEOs and ARM targets that can be detected with ground-based systems decreases as the size of the object decreases ( $H$ increases) because only those 
that approach close to Earth get bright enough to be brighter than a survey's limiting apparent magnitude $\left(V_{\text {limit }}\right)$. To reduce the computation time for the survey simulations we eliminated all the generated objects with MOID (e.g. Gronchi 2005) greater than the maximum distance at which an object with the generated absolute magnitude can be detected by a survey. The maximum distance is (usually) determined by the object having its brightest apparent magnitude $(V)$ when fully illuminated at opposition (i.e. zero phase angle, $\alpha$ ). Thus, we select objects from our two generated populations that satisfy $V(H, \Delta=$ MOID, $\alpha=0)<V_{\text {limit }}$ before running them through the survey simulation. This is equivalent to requiring that MOID $<\Delta(H)$ where $\Delta(H)$ is the $H$-dependent maximum distance at which the object can be detected.

Essentially all the generated NEOs with $H \lesssim 21$ are bright enough to be detected by Pan-STARRS1 if they happen to be at their MOID while near opposition (but most objects will not satisfy these conditions) and the fraction that pass the MOID selection

criterion decreases with the size of the NEO. About $36 \%$ of NEOs satisfy the MOID cut for Pan-STARRS1 at $H \sim 27$, the largest size for our synthetic ARM targets.

Roughly $2-3 \times$ the fraction of ARM targets pass the MOID criterion compared to NEOs at the same absolute magnitude because of the ARM targets's restricted dynamical criteria. The ARM targets's Earth-like, low-eccentricity, low-inclination orbits with $a \sim 1 \mathrm{AU}$ are specifically designed to bring them close to Earth.

\subsection{Pan-STARRS1}

Pan-STARRS1 (e.g. Kaiser 2004; Hodapp et al. 2004) was intended to be a singletelescope prototype for a next-generation survey system Pan-STARRS4 (Kaiser et al. 2002). It is situated in the USA on the summit of Haleakala, Maui, Hawaii (observatory 
code F51) and has been operated by the University of Hawaii since the spring of 2010. Pan-STARRS1 has a $1.8 \mathrm{~m}$ diameter primary mirror with $\sim 7 \mathrm{deg}^{2}$ field of view and can survey $\sim 900 \mathrm{deg}^{2} /$ night for asteroids (i.e. imaged $4 \times /$ night). The Pan-STARRS1 detector system is outfitted with six optical filters $\left(g_{\mathrm{P} 1}, r_{\mathrm{P} 1}, i_{\mathrm{P} 1}, z_{\mathrm{P} 1}, y_{\mathrm{P} 1}, w_{\mathrm{P} 1}\right)$, where the $w_{\mathrm{P} 1}$ filter has a wide bandwidth $\left(\sim g_{\mathrm{P} 1}+r_{\mathrm{P} 1}+i_{\mathrm{P} 1}\right)$ optimized for asteroid detection and the $z_{\mathrm{P} 1}$ filter is a near-IR filter suitable for high sky-background conditions, e.g. surveying close to the Moon.

We simulated the Pan-STARRS1 survey from the beginning of 2014 when the system was nearly 100\% devoted to NEO discovery and the bore sites were arranged mostly near the ecliptic and towards opposition to maximize the likelihood of NEO detection. The bore sites were selected to give a wide berth to the Moon and the galactic plane to avoid regions with scattered moonlight and high stellar sky-plane density. The survey pattern includes a 'sweet spot' component to enhance the detection rate of Potentially Hazardous Objects (PHO), asteroids and comets with $H<22$ and MOID $<0.05$ AU. The sweet spots are patches of sky roughly $60^{\circ}$ to $90^{\circ}$ from the Sun and within $20^{\circ}$ of the ecliptic where the sky-plane density of PHOs is highest (Chesley and Spahr 2004). The average 'transient time interval' (TTI), the time between repeated visits to the same footprint within a night, is $\sim 19$ minutes.

Every morning the image processing pipeline (IPP; Magnier 2006) produced a source list of transient detections that included both real objects and false detections. This list is then processed by the Moving Object Processing System (MOPS; Denneau et al. 2013). MOPS is an integrated system that processes data from per-exposure transient detection source lists to identify moving objects and produces 'tracklets', sets of two or more detections which correspond to the same object. 


\subsection{Pan-STARRS1 survey simulation - PS1*}

We used MOPS to simulate Pan-STARRS1 performance and refer to the simulation as the PS1* survey. MOPS ingests a list of bore sites and times, the size and shape of field of view (FOV), the list of orbital elements and absolute magnitudes of synthetic objects, and determines which of them appear in each field and, if they do so, their apparent magnitudes and apparent rates of motion. MOPS was designed to account for many additional survey factors like identification detection efficiency, camera focal-plane fill-factor, weather, etc., but in our work we accounted for these factors in post-MOPS processing as described in $\S 2.6$.

PS1* uses the actual bore sites visited by Pan-STARRS1 during the time period from 1 January 2014 through 31 May 2015. We selected only bore sites imaged in the $w_{\mathrm{P} 1}$ filter that is optimized for NEO discovery (about $80 \%$ of all NEOs were detected in this band).

$\mathrm{PS}^{*}$ has a fixed limiting magnitude of $V_{\text {limit }}=22.5$, with $100 \%$ detection efficiency for $V<V_{\text {limit }}$ and $0 \%$ for $V \geq V_{\text {limit }}$, dramatically different from the narrow and smooth drop in detection efficiency for the actual system. To compensate for this difference we implemented an ad hoc post-process correction to the $\mathrm{PS}^{*} V$ distribution to ensure that it would match the observed $V$ distribution for real Pan-STARRS1 objects. First, we fit the Pan-STARRS1 survey's asteroids's $V$-distribution to the function

$$
F(V)=\frac{-0.54+0.04 V}{1+\exp ^{(V-21.81) / 0.18}},
$$

where the numerator then provides a measure of the actual increase in number of detected objects as a function of apparent magnitude, but the denominator measures the detection efficiency near the system limiting magnitude:

$$
\epsilon(V)=\frac{1}{\left.1+\exp ^{(V-21.81) / 0.18}\right)} .
$$

We then implemented this efficiency function by randomly rejecting a fraction of detections 
at each limiting magnitude to ensure that our synthetic $V$ distribution matched the actual one.

\subsection{System losses}

The real Pan-STARRS1 survey performance is affected by e.g. weather and system downtime, cosmetic imperfections in the detectors, and by so-called 'trailing losses' in which fast-moving asteroids leave trailed images on the CCD rather than PSF-like detections. Since our PS1* survey used the actual Pan-STARRS1 bore sites there was no need for a weather-related downtime adjustment but we accounted for the following effects that have an impact on the NEO detection efficiency in post-processing:

\subsubsection{Tracklet identification efficiency}

Tracklet identification efficiency is a combination of several factors including the camera's fill factor, the stellar sky-plane density, image processing detection efficiency, etc. The realized Pan-STARRS1 tracklet efficiency even at bright magnitudes is about $75 \%$ due mostly to the fill factor - about $25 \%$ of the Pan-STARRS1 camera focal plane is occupied by gaps between the CCDs, gaps on the CCDs themselves, and inactive pixels. To mimic this loss we randomly deleted $25 \%$ of synthetic detections from the PS1* simulation and required that each remaining tracklet consisted of $\geq 2$ detections. (Pan-STARRS1's standard operating procedures require a minimum of 3 detections for a validated tracklet but not all the detections are necessarily submitted to the Minor Planet Center because some of the detections are contaminated or otherwise adversely affected by focal plane issues. The fraction of 2-detection tracklets actually submitted by Pan-STARRS1 is comparable to the

fraction of 2-detection tracklets generated by our synthetic PS1* technique.) To assess the 
impact of the detection-loss process in the low-statistics, large absolute magnitude regime, we repeated the procedure $1,000 \times$ to evaluate the systematic uncertainties on the final debiased NEO SFD.

\subsubsection{Trailing losses}

An object with high apparent rate of motion, $\omega$, can move across the field of view so fast that it leaves a 'trail' even in short exposures. Trailing spreads the PSF causing a reduction in the per unit area apparent magnitude and signal-to-noise, which leads to the failure to detect many small, fast-moving NEOs (fig. 3). This reduction in tracklet identification efficiency due only to an object's apparent rate of motion is called 'trailing loss' (e.g. Tancredi and Lindgren 1994; Vereš et al. 2012).

Trailing affects synthetic detections twice: (1) - the MOPS PS1* simulation calculates the expected integrated magnitude for synthetic detections but the Pan-STARRS1 system reports the stellar-PSF-fitted magnitude for the trail. The resulting PS1* magnitudes are brighter than they would be in reality given their apparent rate of motion; (2) - the simulation doesn't take into account the actual trailing loss and unlike the real NEOs, faint synthetic detections with high apparent rate of motion remain in the sample.

To account for issue (1) we 'reverse trail-fit' to generate synthetic detections consistent with the real ones generated by the IPP. i.e. we use MOPS to generate the expected apparent magnitude, modify that value in a 'reverse trail-fit', and then determine whether

the trail was detected based on the modified, pseudo-realistic value. We empirically selected a functional form for the 'reverse trail-fit' based on real Pan-STARRS1 detections as illustrated in fig. 4. The data was fit to a logarithmic function of the form

$$
\Delta(\omega)=2.79 \log _{10}(2.07+\omega)-1.28
$$


where $\Delta \equiv V_{I P P}-V_{\text {trail }}, V_{I P P}$ is the stellar-PSF-magnitude calculated by the IPP, and $V_{\text {trail }}$ is a trail-fitted magnitude. The trail fitting algorithm works well for fast NEOs, but fails for objects with rate of motion $\omega<0.7^{\circ} /$ day, where the value of $\Delta$ becomes negative, i.e. that the actual brightness of the object is overestimated. To avoid this issue we assume $\Delta=0$ for all objects slower than $\omega<0.7^{\circ} /$ day during the reverse trail fitting process.

To remove issue (2) we have simulated the trailing losses by removing the affected fast faint synthetic detections (with already corrected magnitudes) from our sample. We have empirically determined the limiting magnitude as a function of the apparent rate of motion for both Pan-STARRS1 and PS1* with a simple linear function such that the effective limiting apparent magnitude is given by

$$
V_{\text {limit }}(\omega)<-0.64 \omega+23.36
$$

where $\omega>1.5^{\circ} /$ day. This limiting magnitude cut was applied post facto to the PS1* simulation after adjusting for the tracklet identification efficiency and 'reversed' trail fit.

As expected, our $\mathrm{PS}^{*}$ simulation shows that there is a dramatic difference between the apparent rates of motion of large and small NEOs and, interestingly also between NEOs and ARM targets at the same size (fig. 5). For example, $1 \mathrm{~km} \mathrm{NEOs}(H \sim 17.5)$ typically can be detected (i.e. are brighter than the survey's $V_{\text {limit }}$ ) at rates of motion of $\lesssim 2^{\circ} /$ day while almost all the small ones with $D \lesssim 100 \mathrm{~m}(\mathrm{H}>27)$ are moving faster than this rate at the time of the discovery. This is a observational selection effect because the surveys are able to detect smaller NEOs only when they make a close approach to Earth which results in their high apparent rates of motion.

The ARM targets' apparent rate of motion is considerably less than the NEOs' apparent rate of motion at the same size because the former's Earth-like orbits ensure that they spend more time in Earth's vicinity (and thus are slower) than generic NEOs. For example, at $H \sim 27$ the average ARM target's apparent rate of motion when detected 
by $\mathrm{PS}^{*}$ is $1.5^{\circ}$ /day, while the average rate of motion for NEOs of the same absolute magnitude is $6.7^{\circ}$ /day. This suggests that objects on the ARM target-like orbits are more likely to be detected by surveys than 'regular' NEOs, which was also noted by Harris and D'Abramo (2015). More importantly they are also easier targets for follow-up astrometric observations that can reduce their orbit fit residuals and ensure they won't get lost.

\subsubsection{NEO identification}

Contemporary asteroid surveys rely on the MPC NEO 'digest' score that is, essentially, the probability that a real tracklet is not a main-belt object (i.e. it may be a NEO) if it can not be associated with a known asteroid. MPC requires that surveys submit and follow-up NEO candidates only with digest score $\geq 65$. Objects with digest score lower than this threshold get submitted as 'incidental astrometry'. MPC assigns them a lower

priority for processing and eventually attempts to find links with previous observations of known objects. If the linking routine fails, tracklets are moved to the isolated tracklet file (ITF), essentially a depository of unlinked and lost detections. Thus, some NEOs 'hide in plain sight' by virtue of being detected but having otherwise mundane locations and rates of motion (e.g. Jedicke 1996).

Fig. 6 shows fraction of synthetic detections with digest score $<65$ per absolute magnitude bin. More than $30 \%$ of the largest and almost $20 \%$ of $\sim 1$ km objects wouldn't be submitted as NEO candidates. However, this is not an issue for larger NEOs because most are already known (e.g. Mainzer et al. 2011a; Harris and D'Abramo 2015). We can also expect that most of the medium-sized NEOs hiding in plain sight will be re-detected with much higher digest score over the course of a multi-year survey. The low scores are critical for smaller objects, where a rapid follow-up is necessary otherwise the orbital uncertainly grows rapidly and they will get lost. Fortunately the majority of small NEOs and ARM 
targets are moving so fast when detected, that their digest score will almost always be $\sim 100$ (fig. 5). Indeed, less than 5\% of synthetic NEOs with $\mathrm{H}>22$ and roughly $7 \%$ of the largest ARM targets have scores this low and will be lost. The fraction is slightly larger for ARM targets compared to NEOs at the same size primarily due to their slower apparent rate of motion.

\section{Results \& discussion}

\subsection{NEO and ARM target detection efficiency}

The survey's NEO detection efficiency, $\epsilon(H)$, is the fraction of objects identified in the simulation as a function of absolute magnitude $H$. We combine discoveries of new objects and detections of known objects into 'identifications' because we make no distinction between known and new NEOs in our simulation. Most of the large objects will be detections, most of the small objects will be discoveries. Fig. 7 shows our simulation is a good representation of reality and the detected synthetic NEOs match the real Pan-STARRS1 detections well in terms of rate of motion, visual magnitude, sky-plane coordinates at discovery and in (a, e, i, H). The realistic simulated survey detection efficiency can then be used as proxy for the actual survey detection efficiency.

We find that the survey's NEO detection efficiency decreases by about 6 orders of magnitude as the cross-sectional area of the objects decreases by $\sim 10^{4} \times$ from $H=18$ to $H=28$ (fig. 8). The NEO detection efficiency in the ARM target size range of $27<H \lesssim 31$

decreases from $\sim 10^{-6}$ to $\sim 10^{-9}$ because these objects are very small and need to be close to Earth to be bright enough to be detected, but are then moving so fast that trailing losses are high (fig. 5).

Interestingly, the ARM target detection efficiency (fig. 8) is $\sim 1000 \times$ higher than the 
NEO detection efficiency at the same absolute magnitude because of the differences in their orbit element distributions — by design, the ARM targets approach close to Earth and spend a relatively long time in Earth's environs so they have a higher probability of being detected. Their slower apparent rate of motion relative to the NEOs accounts for their higher detection efficiency. Even so, our results suggest that only $\sim 0.06 \%$ of the largest ARM targets with $H \sim 27$ are detected annually by PS1*. Their detection efficiency drops $\sim 10^{4} \times$ from $H \sim 27$ to $H \sim 31$ - faster than the number of objects increases as we will show below.

Our conclusion supports the findings of Harris and D'Abramo (2015), even though their definition of ARM-target like bodies is less strict than ours $(\mathrm{D} \sim 10 \mathrm{~m}, \mathrm{MOID} \lesssim 0.03 \mathrm{AU}$ and $\left.v_{\infty}<2.5 \mathrm{~km} / \mathrm{s}\right)$. Their results suggest the current surveys are 1000 times more efficient in detecting these slow moving objects than the average speed bodies and estimate their total population to 2000 .

\subsection{Near-Earth object size-frequency distribution}

By virtue of the similarity of our simulated PS1* survey to the real Pan-STARRS1 the measured detection efficiency (fig. 8) allows us to debias the actual Pan-STARRS1 NEO detections and derive the real NEO SFD. In each H-bin the total number of NEOs (N) is simply the number of NEOs detected by Pan-STARRS1 $\left(n_{P S 1}\right)$ divided by the efficiency of $\mathrm{PS}^{*}(\epsilon)$ measured for the given bin: $N=n_{P S 1} / \epsilon$.

Fig. 9 shows the debiased NEO SFD in comparison with other contemporary models. Our SFD is in excellent agreement with the (Granvik et al. 2016) NEO model that extends the work of Bottke et al. (2002a). All but one of our values in H-bins are within 1- $\sigma$ - interestingly enough the largest disagreement is for $\mathrm{H} \sim 17.5$, where our prediction lies 
directly between the Granvik et al. (2016) and Harris and D'Abramo (2015) SFDs. Our distribution also matches Harris and D'Abramo (2015) within 1- $\sigma$ up to $\mathrm{H}=27$ but our values are systematically below their SFD beyond $H \sim 22$ while we continue to be in excellent agreement with (Granvik et al. 2016) to $H=25$ and (Brown et al. 2002) all the way to $H \sim 30$.

Our derived SFD exhibits smooth variations in the slope as a function of absolute magnitude akin to the Harris and D'Abramo (2015) model. To compare the slope of the distributions we fit our cumulative SFD to a function of the form $N\left(H_{0}\right)=N_{H_{0}} 10^{\alpha\left(H-H_{0}\right)}$ where $N\left(H_{0}\right)$ is the cumulative number of objects with $H<H_{0}, \alpha$ is the slope of the distribution, and $H_{0}$ is the absolute magnitude at which there are $N_{0}$ objects with $H<H_{0}$. We find $N_{13}=8.6 \pm 1.2$ and $\alpha=0.48 \pm 0.02$ for NEOs with $13<H<16$. The slope becomes shallower for NEOs with $16<H<22$ where $N_{16}=353 \pm 34$ and $\alpha=0.33 \pm 0.01$. Around $\mathrm{H}=22$ the slope becomes steep again with $N_{22}=44,400 \pm 4,100$ and $\alpha=0.62 \pm 0.03$ for NEOs with $22<H<27$ which agrees to within 1- $\sigma$ with the Brown et al. (2002, 2013) SFD slope measured for large bolides from infrasound detections. The selection of boundaries in any non-continuous data is difficult, especially for power-law distributions (Newman 2005; Clauset et al. 2009). We empirically identified the $H$-boundaries as the values where the uncertainties on $\alpha$ and $N_{H_{0}}$ exceeded $5 \%$ and $15 \%$ respectively if they were included in the SFD. Note that the uncertainties on our SFD represented by error bars on fig. 9 are statistical only. The systematic uncertainty introduced by detection losses due to the camera's fill factor is depicted by pink lines.

Harris and D'Abramo (2015) speculate that the dip around $19<H<25$ may correspond to a transition in the internal structure of asteroids from rubble piles to monolithic bodies, with its minimum around $D \sim 100 \mathrm{~m}$ representing the weakest objects mostly prone to disruption. The same suggestion was made earlier by e.g. Durda et al. 
(1998); Bottke et al. (2005); O'Brien and Greenberg (2005). Mainzer et al. (2011c) have already shown that there is no observed shift of mean albedo with the diameter of the object (where the transition in the SFD would represent the largest gradient of albedo change), thus the transition must have an actual structural or dynamical cause. Perhaps this transition in SFD points to the size regime where the YORP effects starts to be dominant and increases the spin rate more efficiently. This might lead to a gradual mass loss eventually leading towards more small, fast rotating NEOs (e.g. Bottke et al. 2002b).

Similarly to Harris and D'Abramo (2015) there is an evidence for yet another change in slope for smaller NEOs with $27<H<30$ where our fit yields a steeper slope of $\alpha=0.45 \pm 0.09$ with $N_{27}=16.61 \pm 4.04 \times 10^{6}$. Our SFD is weakly constrained for $H \gtrsim 27$ because of the small number of Pan-STARRS1 detections in this size range and the larger uncertainty on our derived efficiencies.

We used the Harris and D'Abramo (2015) SFD to calculate the NEO detection rates expected over the duration of the survey simply because it covers the absolute magnitude range of our NEO model. Tab. 1 shows the expected rate matches the real data well up to $\mathrm{H} \sim 22$ where it starts to overestimate the number of NEOs compared to real detections by Pan-STARRS1. This is due to the fact that values of Harris and D'Abramo (2015) SFD are systematically above ours for $\mathrm{H} \gtrsim 22$. For example Pan-STARRS1 detected 12 NEOs with $H>27$ during the analyzed time period, while we predict the detection rate of 56 NEOs if we use the SFD of Harris and D'Abramo (2015). On the other hand, Pan-STARRS1 should detect 10 NEOs with $H>27$ when Brown et al. $(2002,2013)$ SFD is used, thus it seems to be a better match for the upper part of NEO SFD than Harris and D'Abramo (2015). 


\subsection{Pan-STARRS1 ARM target discoveries}

Pan-STARRS1 identified 3 ARM target candidates between 1 January 2014 and 31 May 2015 while our PS1* simulation predicted discovery rates of $0.45 \pm 0.13$ /year with the SFD calculated in this work for $27<H<30$. Our prediction lies directly between detection rates based on the Brown et al. (2002) and Harris and D'Abramo (2015) SFDs which give $0.21 \pm 0.02$ and $1.1 \pm 0.3 \mathrm{ARM}$ targets discovered per year respectively (tab. 2). The detection rate of $<2 /$ year seems to be robust since PS1 has detected a total of 9 ARM targets since operations began in early 2010. A simple extrapolation of the Bottke et al. (2002a) NEO SFD to the ARM target absolute magnitude range would predict $120 \times$ more than were actually identified, but this extrapolation is unwarranted given the model's applicable range of $H<22$.

We used a maximum-likelihood (ML) fit to determine the slope $(\alpha)$ of the SFD for the 3 Pan-STARRS1 ARM targets to avoid the numerical issues of binning them in $0.5 \mathrm{mag}$ $H$-bins and thereby losing resolution on the calculated absolute magnitudes. To do so we first fit the ARM target detection efficiency $\left(\epsilon_{A R M}\right.$; fig 8$)$ of our PS1* simulation and found $\log _{10} \epsilon_{A R M}=(-2.96 \pm 0.17)-(1.04 \pm 0.08) \times(H-27)$ for $27<H<31$. The ML

fit yielded an ARM target SFD $\propto 10^{\alpha H}$ with $\alpha=0.40_{-0.45}^{+0.33}$, suggestive of a shallow and non-equilibrium SFD but consistent with almost anything.

The ARM targets as a dynamically restricted subset of NEOs with low $\Delta v$ may be the proposed 'undetected' population of low speed lunar projectiles noted by Ito and Malhotra (2010) from lunar cratering records. Alas, several works (Gladman et al. 1995; Gaskin et al. 1998, e.g.) suggest that some fraction of lunar ejecta will return to the Earth-Moon system within several thousand to million years. Some ARM targets might also be remnants from NEOs tidally disrupted during close encounters with Earth which tend to return to Earth's environs and eventually re-impact Earth within $\sim 10^{4}$ years (Schunová et al. 2014). Finally, 
a small percentage are man made such as Apollo rocket boosters (Chodas 2013).

\subsection{Follow-up observation windows}

One issue with current Pan-STARRS1 discoveries is the lack of astrometric follow-up observations for fast and/or faint objects. While Pan-STARRS1 can self follow-up it is better suited to rapidly surveying the sky with its wide-field camera than targeting specific objects. This leads inevitably to the loss of many potentially interesting NEOs because there is usually only limited time available for followup observations aimed at securing the object's orbit and obtaining measurements suitable for physical characterization (Mommert et al. 2016; Thirouin et al. 2016).

Here we used synthetic NEOs and ARM targets detected by our PS1* survey to assess the time windows suitable for follow up observations with the CFHT (Tholen 2001), Pan-STARRS1 and NASA IRTF telescopes and also for the Arecibo and Goldstone radars (Benner et al. 2015).

For each detected synthetic PS1* object we calculated an ephemeris for 100 days after the 'discovery' epoch to determine how long it would remain in the followup site's observing window in terms of RA, declination, maximum altitude during the night/day and $V_{\text {lim }}$, or how long it will be within the SNR range for the radar facilities. We assumed the minimum required radar $\mathrm{SNR}=10$ for a detection ${ }^{4}$.

While virtually all (>99\%) synthetic NEOs detected by PS1* were accessible for follow-up by Pan-STARRS1 and CFHT due to their fainter limiting magnitudes, many never reached sufficient brightness or SNR necessary for follow-up with IRTF, Arecibo, or

\footnotetext{
${ }^{4}$ P.Chodas (NASA JPL) - personal communication
} 
Goldstone (fig. 10). The fraction of objects accessible for followup decreases with increasing $H$ for IRTF to $H \sim 22$ while it remains flat for both radar systems in the same range. The opportunity for followup increases for all three facilities for $H \gtrsim 22$ due to observation selection effects - small objects in this absolute magnitude range need to be close enough to be detected by Pan-STARRS1and, by the virtue of their proximity, some of them become brighter than IRTF's limiting magnitude and also reach the limiting SNR of both radars.

As expected IRTF/SPEX (Rayner et al. 2003) has the shortest average observation window due to its lower $V_{l i m}=18.5$ (fig. 11). It is necessary to act quickly if one wants obtain a physical characterization of small NEAs and ARM targets with this instrument because these objects will be out of its reach within several days. Follow up windows get progressively longer for Pan-STARRS1 and CFHT due to their deeper $V_{\text {lim }}$. Pan-STARRS1 is able to detect objects up to $\mathrm{V}=22.5$ in $w_{\mathrm{P} 1}$-band during $45 \mathrm{~s}$ exposure, while CFHT's MegaCam (Boulade et al. 2003) is able to reach 24.0 mag in r-band during a $60 \mathrm{~s}$ exposure. The average windows for small NEOs are only $~ 20$ days duration for these instruments but this might be enough to obtain astrometric and photometric measurements necessary for orbit determination and physical characterization. Both CFHT and Pan-STARRS1 are equipped with cameras with large FOV ( $1 \mathrm{deg}^{2}$ and $\sim 7 \mathrm{deg}^{2}$ respectively), which makes them ideal for 'chasing' fast close objects.

ARM targets are very difficult objects to follow-up - out of the complete set $(27<H<31)$ of the synthetic ARM targets detected by PS1* , only $24 \%$ were bright enough to reach the $\mathrm{V}_{\text {lim }}$ of IRTF and those were observable only for an average of 2.4 days. Pan-STARRS1 and CFHT would be more successful since all synthetic ARM targets were available for follow-up with these facilities and the average observing windows were 26.5 and 44.5 days respectively. Figure 11 shows that ARM targets have in average 2-3 times longer windows as NEOs in the same H-range no matter what facility is used which is again 
accountable to their much smaller apparent motion compared to that of NEOs in the same size.

The average radar windows for both both populations are typically shorter compared to the optical telescopes, which can be also accounted to the different geographic position of these facilities. In average $48.4 \%$ and $41.0 \%$ of ARM targets were available for follow-up with Arecibo and Goldstone radars respectively with average observing window of 10.5 and 9.5 days.

\section{Conclusions}

Our PS1* simulation is a good proxy of the actual Pan-STARRS1 survey based on the fact that it reproduces very well the $(\mathrm{a}, \mathrm{e}, \mathrm{i}, \mathrm{H})$ - distribution and visual magnitudes and apparent rate of motion of the real NEOs detected by Pan-STARRS1 .

We used our normalized PS1* annual detection efficiency to debias the Pan-STARRS1 system's identified NEOs as a function of absolute magnitude. The resulting $H$ distribution is an excellent match to the new Granvik et al. (2016) NEO model and its shape is akin to the Harris and D'Abramo (2015) distribution for $H<22$ where it exhibits multiple transitions between shallow and steep slopes.

Our best fit to the cumulative SFD yields $\alpha=0.48 \pm 0.02$ for NEOs with $13<H<16$, $\alpha=0.33 \pm 0.01$ for NEOs with $16<H<22$ and finally a steep slope of $\alpha=0.62 \pm 0.03$ for the small NEO population with $22<H<27$ which agrees within $1-\sigma$ with the Brown et al. (2002, 2013) SFD slope measured for large bolides from infrasound detections. Even smaller NEOs with $H>27$ exhibit a possible turn to a shallower slope where our fit yields $\alpha=0.45 \pm 0.09$.

Our maximum-likelihood fit to the Pan-STARRS1 ARM target SFD yielded 
$\alpha=0.40_{-0.45}^{+0.33}$, consistent with the entire range of slopes for the known NEO population.

We show that the windows for follow up observations of small $(\mathrm{H} \gtrsim 22)$ NEOs and ARM targets with NASA IRTF telescope and Arecibo and Goldstone radars are extremely short - in order of several days and the CFHT's MegaCam and Pan-STARRS1 would be more successful in obtaining astrometric and/or photometric observations of objects across the whole inspected H-range. Thus we recommend that procedures for fast response should be implemented in order to measure physical characteristics, such as rotation rate, shape, and spectra of small Earth-approaching objects. 


\section{Acknowledgments}

We thank Paul Chodas, Donald Yeomans and Steve Chesley from NASA's NEO office at the Jet Propulsion Laboratory for defining the ARM target population and helpful discussions about the small NEO population; Scott Stuart, MIT Lincoln Laboratory, for his insights on measuring the ARM targets' size-frequency distribution; the reviewers for many helpful comments and extensive discussion; and Robert Weryk from the IfA Unversity Hawaii for his thorough revision of the manuscript. Eva Lilly thanks her newborn daughter Elizabeth for being a good baby so she could finish the paper in peace.

The Pan-STARRS1 Surveys (PS1) have been made possible through contributions of the Institute for Astronomy, the University of Hawaii, the Pan-STARRS Project Office, the Max-Planck Society and its participating institutes, the Max Planck Institute for Astronomy, Heidelberg and the Max Planck Institute for Extraterrestrial Physics, Garching, The Johns Hopkins University, Durham University, the University of Edinburgh, Queen?s University Belfast, the Harvard-Smithsonian Center for Astrophysics, the Las Cumbres Observatory Global Telescope Network Incorporated, the National Central University of Taiwan, the Space Telescope Science Institute, the National Aeronautics and Space Administration under Grant Nos. NNX08AR22G, NNX12AR65G, and NNX14AM74G issued through the Planetary Science Division of the NASA Science Mission Directorate, the National Science Foundation under Grant No. AST-1238877, the University of Maryland, and Eotvos Lorand University (ELTE) and the Los Alamos National Laboratory. 


\section{REFERENCES}

Abell, P., Mazanek, D., Reeves, D., Naasz, B. and Cichy, B. (2015), NASA's Asteroid Redirect Mission (ARM), in 'AAS/Division for Planetary Sciences Meeting Abstracts', Vol. 47 of AAS/Division for Planetary Sciences Meeting Abstracts, p. 312.06 .

Andreev, G. V., Goldin, V. V. and Vasilyev, N. V. (1993), '1908 Tunguska Event: Energy, Orbit, Composition, Origin', LPI Contributions 810, 11.

Benner, L. A. M., Busch, M. W., Giorgini, J. D., Taylor, P. A. and Margot, J.-L. (2015), Radar Observations of Near-Earth and Main-Belt Asteroids, in P. Michel, F. E. DeMeo and W. F. Bottke, eds, 'Asteroids IV', University of Arizona Press, pp. $165-182$.

Borovička, J., Spurný, P., Brown, P., Wiegert, P., Kalenda, P., Clark, D. and Shrbený, L. (2013), 'The trajectory, structure and origin of the Chelyabinsk asteroidal impactor', Nature 503, 235-237.

Bottke, J. W. F., Vokrouhlický, D., Rubincam, D. P. and Broz, M. (2002b), The Effect of Yarkovsky Thermal Forces on the Dynamical Evolution of Asteroids and Meteoroids, in W. Bottke, A. Cellino, P. Paolicchi and R. P. Binzel, eds, 'Asteroids III', University of Arizona Press, pp. 395-408.

Bottke, W. F., Durda, D. D., Nesvorný, D., Jedicke, R., Morbidelli, A., Vokrouhlický, D. and Levison, H. F. (2005), 'Linking the collisional history of the main asteroid belt to its dynamical excitation and depletion', Icarus 179, 63-94.

Bottke, W. F., Morbidelli, A., Jedicke, R., Petit, J.-M., Levison, H. F., Michel, P. and Metcalfe, T. S. (2002a), 'Debiased Orbital and Absolute Magnitude Distribution of the Near-Earth Objects', Icarus 156, 399-433. 
Boulade, O., Charlot, X., Abbon, P., Aune, S., Borgeaud, P., Carton, P.-H., Carty, M., Da Costa, J., Deschamps, H., Desforge, D., Eppellé, D., Gallais, P., Gosset, L., Granelli, R., Gros, M., de Kat, J., Loiseau, D., Ritou, J.-., Roussé, J. Y., Starzynski, P., Vignal, N. and Vigroux, L. G. (2003), MegaCam: the new Canada-France-Hawaii Telescope wide-field imaging camera, in M. Iye and A. F. M. Moorwood, eds, 'Instrument Design and Performance for Optical/Infrared Ground-based Telescopes', Vol. 4841 of Proc. SPIE, pp. 72-81.

Brown, P. G., Assink, J. D., Astiz, L., Blaauw, R., Boslough, M. B., Borovička, J., Brachet, N., Brown, D., Campbell-Brown, M., Ceranna, L., Cooke, W., de Groot-Hedlin, C., Drob, D. P., Edwards, W., Evers, L. G., Garces, M., Gill, J., Hedlin, M., Kingery, A., Laske, G., Le Pichon, A., Mialle, P., Moser, D. E., Saffer, A., Silber, E., Smets, P., Spalding, R. E., Spurný, P., Tagliaferri, E., Uren, D., Weryk, R. J., Whitaker, R. and Krzeminski, Z. (2013), 'A 500-kiloton airburst over Chelyabinsk and an enhanced hazard from small impactors', Nature 503, 238-241.

Brown, P., Spalding, R. E., ReVelle, D. O., Tagliaferri, E. and Worden, S. P. (2002), 'The flux of small near-Earth objects colliding with the Earth', Nature 420, 294-296.

Brown, P., Wiegert, P., Clark, D. and Tagliaferri, E. (2016), 'Orbital and physical characteristics of meter-scale impactors from airburst observations', Icarus 266, 96-111.

Chesley, S. R. and Spahr, T. B. (2004), Earth impactors: orbital characteristics and warning times, in M. J. S. Belton, T. H. Morgan, N. H. Samarasinha, \& D. K. Yeomans, ed., 'Mitigation of Hazardous Comets and Asteroids', pp. 22-+.

Chodas, P. (2013), Estimated ARM candidate target population and projected discovery 
rate of ARM candidates., in 'Presented at Target NEO 2 Workshop, Washington, DC, July 9, 2013'.

Clauset, A., Shalizi, C. R. and Newman, M. E. J. (2009), 'Power-law distributions in empirical data', SIAM Review 51(4), 661-703.

Denneau, L., Jedicke, R., Grav, T., Granvik, M., Kubica, J., Milani, A., Vereš, P., Wainscoat, R., Chang, D., Pierfederici, F., Kaiser, N., Chambers, K. C., Heasley, J. N., Magnier, E. A., Price, P. A., Myers, J., Kleyna, J., Hsieh, H., Farnocchia, D., Waters, C., Sweeney, W. H., Green, D., Bolin, B., Burgett, W. S., Morgan, J. S., Tonry, J. L., Hodapp, K. W., Chastel, S., Chesley, S., Fitzsimmons, A., Holman, M., Spahr, T., Tholen, D., Williams, G. V., Abe, S., Armstrong, J. D., Bressi, T. H., Holmes, R., Lister, T., McMillan, R. S., Micheli, M., Ryan, E. V., Ryan, W. H. and Scotti, J. V. (2013), 'The Pan-STARRS Moving Object Processing System', PASP 125, 357-395.

Durda, D. D., Greenberg, R. and Jedicke, R. (1998), 'Collisional Models and Scaling Laws: A New Interpretation of the Shape of the Main-Belt Asteroid Size Distribution', Icarus 135, 431-440.

Gaskin, J., Zook, H. and Liou, J.-C. (1998), Lunar Ejecta Trajectory Study Utilizing a Gauss-Radau Integrator, in 'Lunar and Planetary Science Conference', Vol. 29 of Lunar and Planetary Science Conference.

Gladman, B. J., Burns, J. A., Duncan, M. J. and Levison, H. F. (1995), 'The dynamical evolution of lunar impact ejecta.', Icarus 118, 302-321.

Granvik, M., Morbidelli, A., Jedicke, R., Bolin, B., Bottke, W. F., Beshore, E., Vokrouhlický, D., Delbò, M. and Michel, P. (2016), 'Super-catastrophic disruption of asteroids at small perihelion distances', Nature 530, 303-306. 
Greenstreet, S., Ngo, H. and Gladman, B. (2012), 'The orbital distribution of Near-Earth Objects inside Earth's orbit', Icarus 217, 355-366.

Gronchi, G. F. (2005), 'An Algebraic Method to Compute the Critical Points of the Distance Function Between Two Keplerian Orbits', Celestial Mechanics and Dynamical Astronomy 93, 295-329.

Harris, A. W. and D'Abramo, G. (2015), 'The population of near-Earth asteroids', Icarus 257, 302-312.

Hodapp, K. W., Kaiser, N., Aussel, H., Burgett, W., Chambers, K. C., Chun, M., Dombeck, T., Douglas, A., Hafner, D., Heasley, J., Hoblitt, J., Hude, C., Isani, S., Jedicke, R., Jewitt, D., Laux, U., Luppino, G. A., Lupton, R., Maberry, M., Magnier, E., Mannery, E., Monet, D., Morgan, J., Onaka, P., Price, P., Ryan, A., Siegmund, W., Szapudi, I., Tonry, J., Wainscoat, R. and Waterson, M. (2004), 'Design of the Pan-STARRS telescopes', Astronomische Nachrichten 325, 636-642.

Ito, T. and Malhotra, R. (2010), 'Asymmetric impacts of near-Earth asteroids on the Moon', Astronomy and Astrophysics 519, A63.

Ivanov, B. A. (2006), 'Earth/Moon impact rate comparison: Searching constraints for lunar secondary/primary cratering proportion', Icarus 183, 504-507.

Jedicke, R. (1996), 'Detection of Near Earth Asteroids Based Upon Their Rates of Motion', AJ 111, 970.

Kaiser, N. (2004), Pan-STARRS: a wide-field optical survey telescope array, in J. M. Oschmann, Jr., ed., 'Society of Photo-Optical Instrumentation Engineers (SPIE) Conference Series', Vol. 5489 of Society of Photo-Optical Instrumentation Engineers (SPIE) Conference Series, pp. 11-22. 
Kaiser, N., Aussel, H., Burke, B. E., Boesgaard, H., Chambers, K., Chun, M. R., Heasley, J. N., Hodapp, K.-W., Hunt, B., Jedicke, R., Jewitt, D., Kudritzki, R., Luppino, G. A., Maberry, M., Magnier, E., Monet, D. G., Onaka, P. M., Pickles, A. J., Rhoads, P. H. H., Simon, T., Szalay, A., Szapudi, I., Tholen, D. J., Tonry, J. L., Waterson, M. and Wick, J. (2002), Pan-STARRS: A Large Synoptic Survey Telescope Array, in J. A. Tyson and S. Wolff, eds, 'Society of Photo-Optical Instrumentation Engineers (SPIE) Conference Series', Vol. 4836 of Society of Photo-Optical Instrumentation Engineers (SPIE) Conference Series, pp. 154-164.

Magnier, E. (2006), The Pan-STARRS PS1 Image Processing Pipeline, in 'The Advanced Maui Optical and Space Surveillance Technologies Conference'.

Mainzer, A., Bauer, J., Grav, T., Masiero, J., Cutri, R. M., Dailey, J., Eisenhardt, P., McMillan, R. S., Wright, E., Walker, R., Jedicke, R., Spahr, T., Tholen, D., Alles, R., Beck, R., Brandenburg, H., Conrow, T., Evans, T., Fowler, J., Jarrett, T., Marsh, K., Masci, F., McCallon, H., Wheelock, S., Wittman, M., Wyatt, P., DeBaun, E., Elliott, G., Elsbury, D., Gautier, IV, T., Gomillion, S., Leisawitz, D., Maleszewski, C., Micheli, M. and Wilkins, A. (2011a), 'Preliminary Results from NEOWISE: An Enhancement to the Wide-field Infrared Survey Explorer for Solar System Science', ApJ 731, 53.

Mainzer, A., Grav, T., Bauer, J., Masiero, J., McMillan, R. S., Cutri, R. M., Walker, R., Wright, E., Eisenhardt, P., Tholen, D. J., Spahr, T., Jedicke, R., Denneau, L., DeBaun, E., Elsbury, D., Gautier, T., Gomillion, S., Hand, E., Mo, W., Watkins, J., Wilkins, A., Bryngelson, G. L., Del Pino Molina, A., Desai, S., Gómez Camus, M., Hidalgo, S. L., Konstantopoulos, I., Larsen, J. A., Maleszewski, C., Malkan, M. A., Mauduit, J.-C., Mullan, B. L., Olszewski, E. W., Pforr, J., Saro, A., Scotti, J. V. 
and Wasserman, L. H. (2011c), 'NEOWISE Observations of Near-Earth Objects: Preliminary Results', ApJ 743, 156.

Mainzer, A., Grav, T., Masiero, J., Bauer, J., Wright, E., Cutri, R. M., McMillan, R. S., Cohen, M., Ressler, M. and Eisenhardt, P. (2011b), 'Thermal Model Calibration for Minor Planets Observed with Wide-field Infrared Survey Explorer/NEOWISE', ApJ 736, 100.

Mommert, M., Farnocchia, D., Hora, J. L., Chesley, S. R., Trilling, D. E., Chodas, P. W., Mueller, M., Harris, A. W., Smith, H. A. and Fazio, G. G. (2014b), 'Physical Properties of Near-Earth Asteroid 2011 MD', ApJ 789, L22.

Mommert, M., Hora, J. L., Farnocchia, D., Chesley, S. R., Vokrouhlický, D., Trilling, D. E., Mueller, M., Harris, A. W., Smith, H. A. and Fazio, G. G. (2014a), 'Constraining the Physical Properties of Near-Earth Object 2009 BD', ApJ 786, 148.

Mommert, M., Trilling, D. E., Borth, D., Jedicke, R., Butler, N., Reyes-Ruiz, M., Pichardo, B., Petersen, E., Axelrod, T. and Moskovitz, N. (2016), 'First Results from the Rapid-response Spectrophotometric Characterization of Near-Earth Objects using UKIRT', AJ 151, 98.

Newman, M. E. J. (2005), 'Power laws, pareto distributions and zipf's law', Contemporary Physics 46(5), 323-351.

URL: http://dx.doi.org/10.1080/00107510500052444

O'Brien, D. P. and Greenberg, R. (2005), 'The collisional and dynamical evolution of the main-belt and NEA size distributions', Icarus 178, 179-212.

Pravec, P. and Harris, A. W. (2000), 'Fast and Slow Rotation of Asteroids', Icarus 148, 12-20. 
Rayner, J. T., Toomey, D. W., Onaka, P. M., Denault, A. J., Stahlberger, W. E., Vacca, W. D., Cushing, M. C. and Wang, S. (2003), 'SpeX: A Medium-Resolution 0.8-5.5 Micron Spectrograph and Imager for the NASA Infrared Telescope Facility', PASP 115, 362-382.

Scheeres, D. J., Hartzell, C. M., Sánchez, P. and Swift, M. (2010), 'Scaling forces to asteroid surfaces: The role of cohesion', Icarus 210, 968-984.

Schunová, E., Jedicke, R., Walsh, K. J., Granvik, M., Wainscoat, R. J. and Haghighipour, N. (2014), 'Properties and evolution of NEO families created by tidal disruption at Earth', ArXiv e-prints .

Shoemaker, E. M. and Helin, E. F. (1978), Earth-approaching asteroids as targets for exploration, in D. Morrison and W. C. Wells, eds, 'NASA Conference Publication', Vol. 2053 of NASA Conference Publication, pp. 245-256.

Tancredi, G. (1998), 'An Asteroid in a Earth-Like Orbit', Celestial Mechanics and Dynamical Astronomy 69, 119-132.

Tancredi, G. and Lindgren, M. (1994), 'Searching for comets encountering Jupiter: First campaign', Icarus 107, 311-321.

Thirouin, A., Moskovitz, N., Binzel, R. P., Christensen, E., DeMeo, F. E., Person, M. J., Polishook, D., Thomas, C. A., Trilling, D., Willman, M., Hinkle, M., Burt, B., Avner, D. and Aceituno, F. J. (2016), 'The Mission Accessible Near-Earth Objects Survey (MANOS): first photometric results', ArXiv e-prints .

Tholen, D. J. (2001), NEO Follow-up at Mauna Kea Observatory, in S. Isobe and Y. Asakuro, eds, 'International Workshop on Collaboration and Coordination among NEO Observers and Orbital Computers held at Kurshiki City Art Museum, Japan 
from October 23 to 26, 2001 organized by Japan Spaceguard Association; edited by Syuzo Isobe and Yoshifusa Asakuro, pp.49-58', pp. 49-58.

Vereš, P., Jedicke, R., Denneau, L., Wainscoat, R., Holman, M. J. and Lin, H.-W. (2012), 'Improved Asteroid Astrometry and Photometry with Trail Fitting', PASP 124, 1197-1207. 
Table 1. NEO detection efficiency $(\epsilon)$ and predicted number of detections for the simulated PS1* survey assuming the SFD from Harris and D'Abramo (2015), and the actual number of detections for the Pan-STARRS1 survey for the time period 2014 January 1 to 2015 May 31.

\begin{tabular}{cccc}
\hline \hline & & \\
$\mathrm{H}_{V}$ range $(\mathrm{mag})$ & $\epsilon \pm \Delta \epsilon\left(\mathrm{PS}^{*}\right)$ & $\mathrm{N} \pm \Delta \mathrm{N}\left(\mathrm{PS} 1^{*}\right)$ & $\mathrm{N}(\mathrm{Pan}-\mathrm{STARRS} 1)$ \\
\hline & & \\
$13.0-14.0$ & $6.23 \pm 0.25 \times 10^{-1}$ & $6.3 \pm 0.3$ & 8 \\
$14.0-15.0$ & $6.13 \pm 0.25 \times 10^{-1}$ & $24.9 \pm 1.0$ & 21 \\
$15.0-16.0$ & $6.10 \pm 0.25 \times 10^{-1}$ & $87.2 \pm 3.5$ & 64 \\
$16.0-17.0$ & $4.88 \pm 0.16 \times 10^{-1}$ & $152.6 \pm 5.1$ & 122 \\
$17.0-18.0$ & $3.26 \pm 0.10 \times 10^{-1}$ & $233.3 \pm 9.4$ & 194 \\
$18.0-19.0$ & $2.03 \pm 0.01 \times 10^{-1}$ & $330.7 \pm 21.5$ & 290 \\
$19.0-20.0$ & $1.01 \pm 0.00 \times 10^{-1}$ & $318.6 \pm 30.6$ & 284 \\
$20.0-21.0$ & $4.77 \pm 0.20 \times 10^{-2}$ & $297.4 \pm 41.2$ & 248 \\
$21.0-22.0$ & $1.63 \pm 0.08 \times 10^{-2}$ & $215.7 \pm 41.6$ & 174 \\
$22.0-23.0$ & $5.06 \pm 0.32 \times 10^{-3}$ & $212.6 \pm 54.1$ & 135 \\
$23.0-24.0$ & $1.28 \pm 0.12 \times 10^{-3}$ & $257.9 \pm 81.8$ & 137 \\
$24.0-25.0$ & $1.51 \pm 0.28 \times 10^{-4}$ & $182.9 \pm 70.9$ & 118 \\
$25.0-26.0$ & $3.25 \pm 0.90 \times 10^{-5}$ & $185.6 \pm 80.3$ & 74 \\
$26.0-27.0$ & $2.00 \pm 1.00 \times 10^{-6}$ & $48.3 \pm 28.9$ & 42 \\
$27.0-28.0$ & $5.97 \pm 1.65 \times 10^{-7}$ & $43.4 \pm 18.8$ & 8 \\
$28.0-29.0$ & $7.00 \pm 2.65 \times 10^{-8}$ & $10.9 \pm 5.5$ & 3 \\
$29.0-30.0$ & $3.34 \pm 3.30 \times 10^{-9}$ & $1.5 \pm 0.0$ & 1 \\
\hline
\end{tabular}


Table 2. Detection efficiency $(\epsilon)$ and predicted number of ARM target detections for the simulated PS1* survey assuming the SFD from Harris and D'Abramo (2015), and the actual number of detections for the Pan-STARRS1 survey for the time period 2014 January 1 to 2015 May 31. Harris and D'Abramo (2015) SFD does not contain data for $30.5<H<31.0$.

\begin{tabular}{cccc}
\hline \hline & & \\
$\mathrm{H}_{V}$ range $(\mathrm{mag})$ & $\epsilon \pm \Delta \epsilon\left(\mathrm{PS}^{*}\right)$ & $\mathrm{N} \pm \Delta \mathrm{N}\left(\mathrm{PS} 1^{*}\right)$ & $\mathrm{N}(\mathrm{Pan}-\mathrm{STARRS} 1)$ \\
\hline & $\times 10^{-10}$ & $\times 10^{-2}$ & \\
\hline $27.0-27.5$ & $191.4 \pm 33.3$ & $52.3 \pm 25.4$ & 1 \\
$27.5-28.0$ & $55.1 \pm 12.6$ & $25.0 \pm 12.8$ & 2 \\
$28.0-28.5$ & $27.1 \pm 7.2$ & $20.1 \pm 10.7$ & 0 \\
$28.5-29.0$ & $2.9 \pm 2.1$ & $2.4 \pm 2.0$ & 0 \\
$29.0-29.5$ & $1.7 \pm 0.8$ & $3.4 \pm 2.4$ & 0 \\
$29.5-30.0$ & $0.6 \pm 0.3$ & $1.5 \pm 1.2$ & 0 \\
$30.0-30.5$ & $0.1 \pm 0.1$ & $0.4 \pm 0.4$ & 0 \\
$30.5-31.0$ & $0.1 \pm 0.1$ & - \pm- & 0 \\
\hline
\end{tabular}



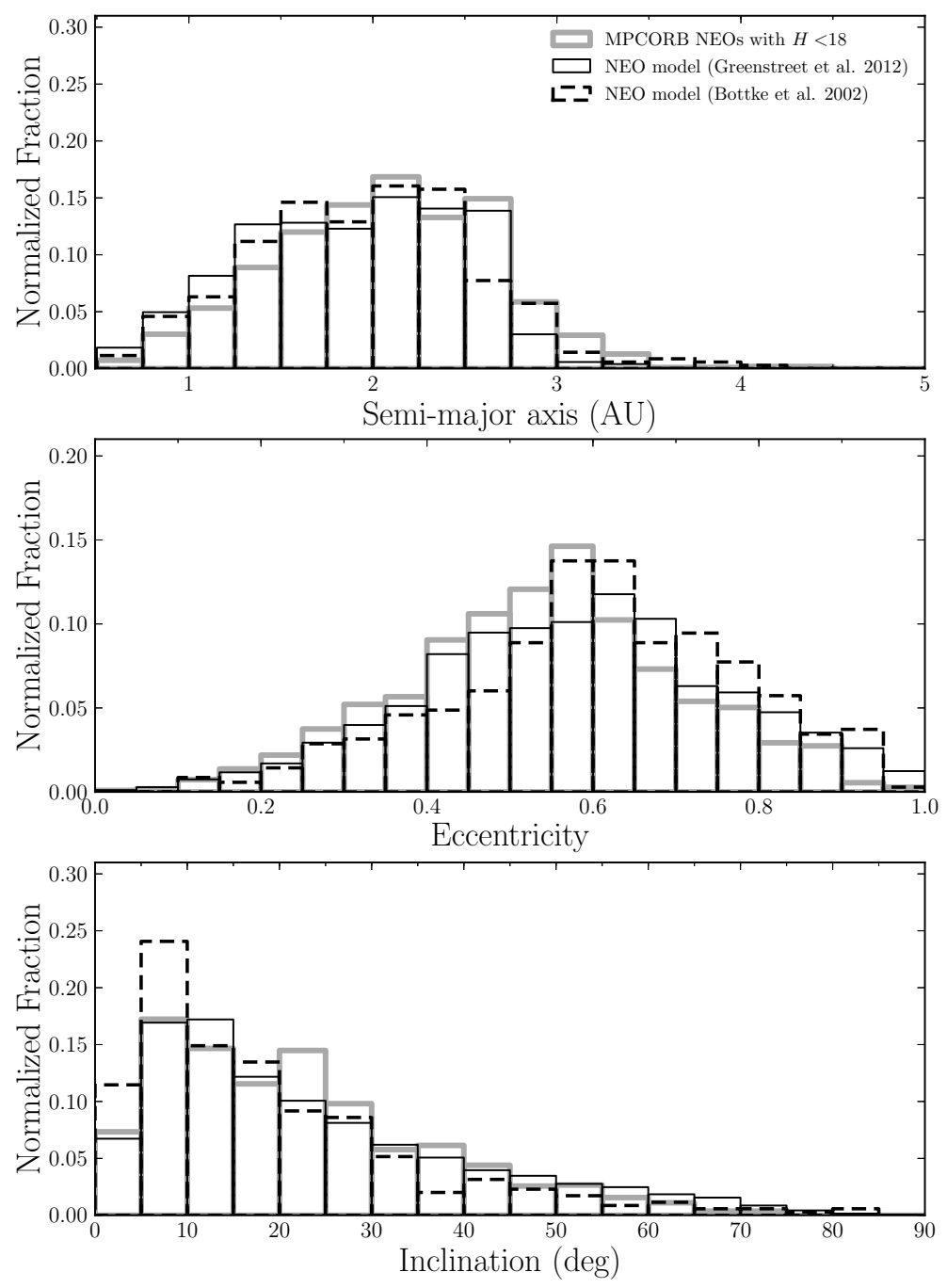

Fig. 1.- Semi-major axis, eccentricity and inclination distributions of known NEOs with $H<18$ and the predicted distributions from the Bottke et al. (2002a) and Greenstreet et al. (2012) models. 

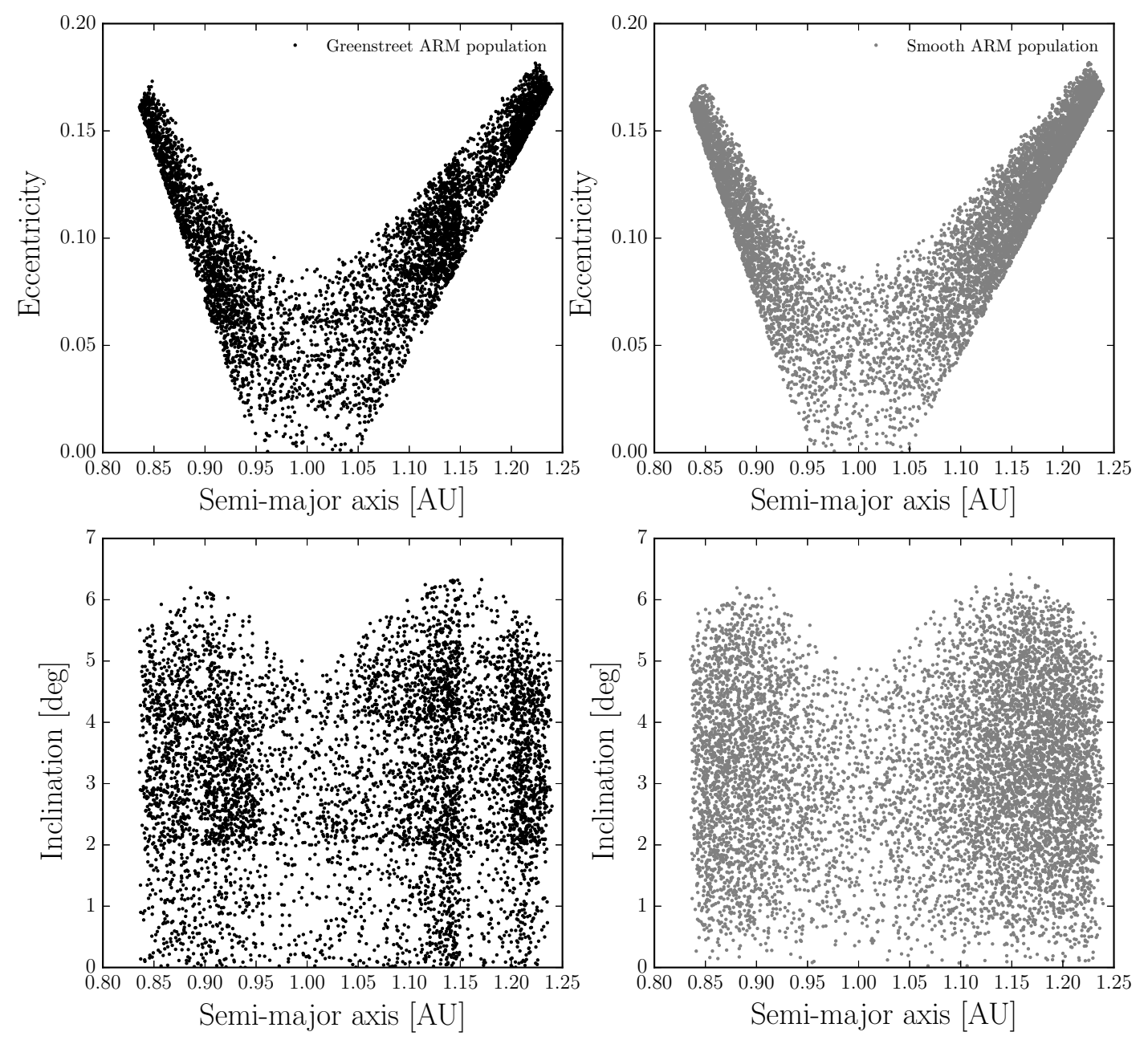

Fig. 2.- (top) Eccentricity vs. semi-major axis and (bottom) inclination vs. semi-major axis for synthetic ARM targets generated according to the raw (left) and smoothed (right) Greenstreet et al. (2012) NEO model (§2.2). Note the limited range of each element and the disappearance of bin edges in the smoothed population. 

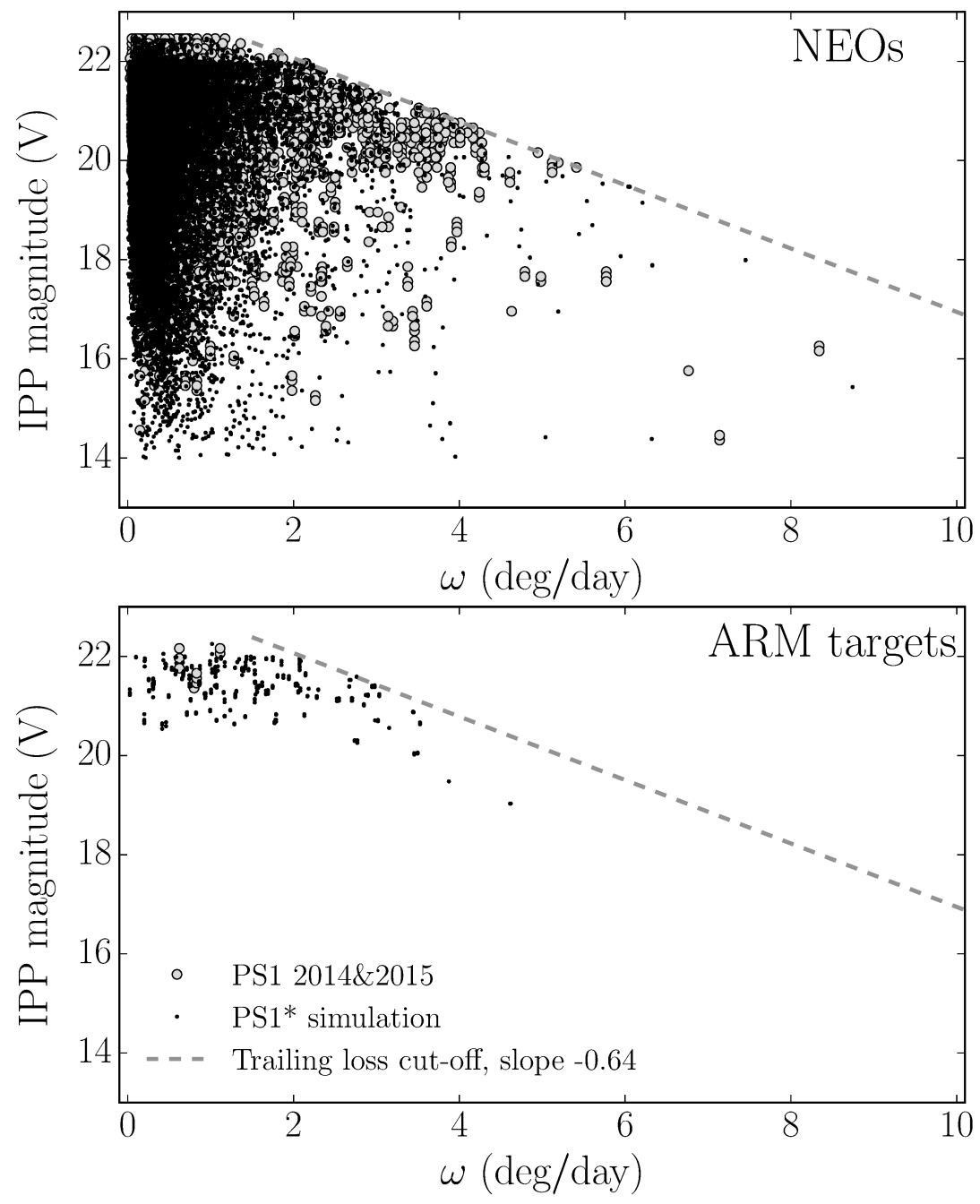

Fig. 3.- $V$ magnitude measured by the IPP vs. rate of motion $\omega$ of real and synthetic NEOs (top) and real and synthetic ARM targets (bottom) detected with Pan-STARRS1 and in our PS1* simulation. The dashed gray line represents the empirical trailing loss limit above which asteroids are too faint to be reliably detected. Trailing losses begin at about $0.5^{\circ}$ day for Pan-STARRS1 and we applied the same trailing loss limit in our PS1* simulation. Some Pan-STARRS1 discoveries do appear above the loss limit line due to improvements in MOPS operations but we applied the same cuts to ensure a consistent comparison between our simulation and the actual data set. 


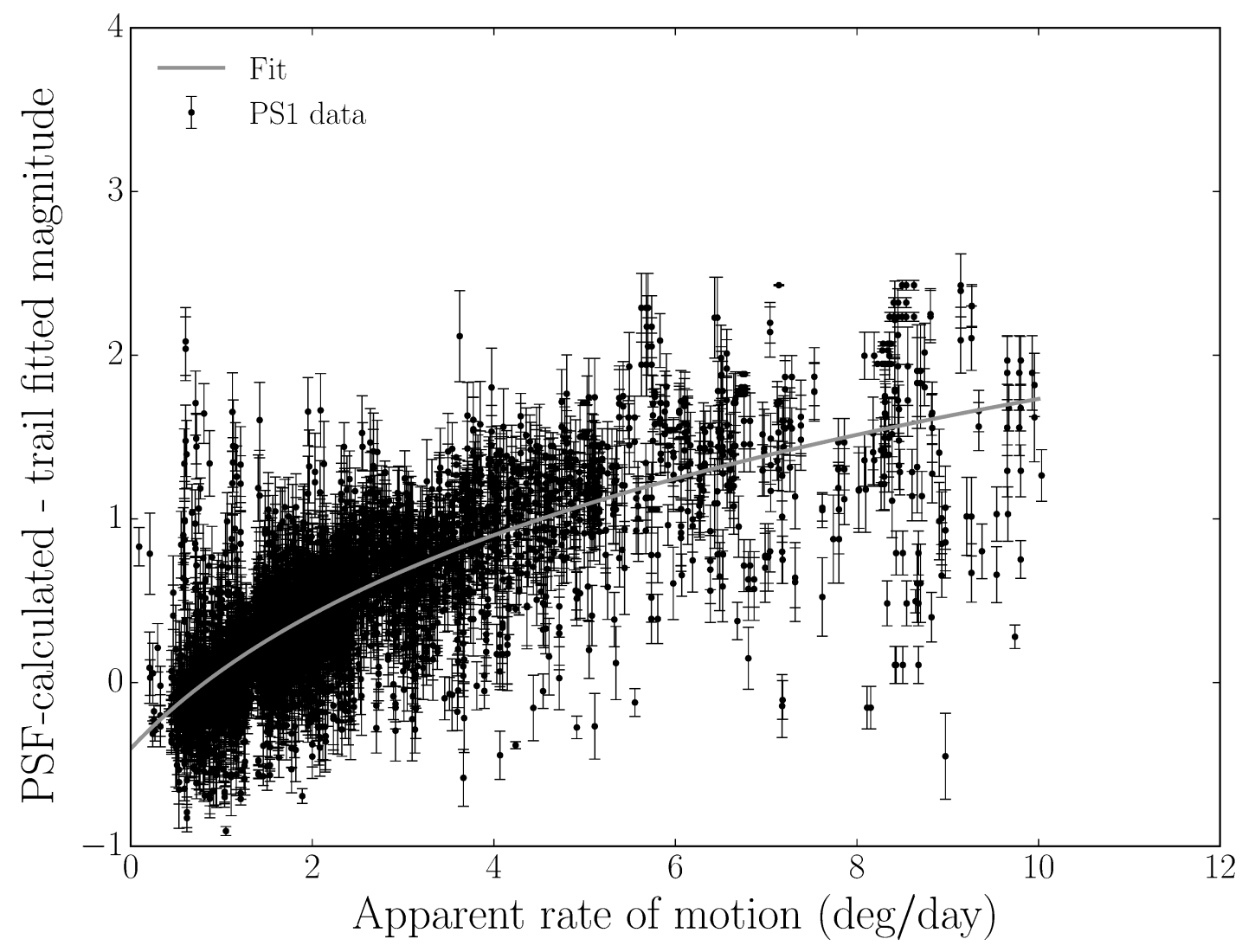

Fig. 4.- Difference $(\Delta)$ between the Pan-STARRS1 IPP's PSF-calculated and our trailfitted magnitudes for real detections as a function of their apparent motion $(\omega)$. 

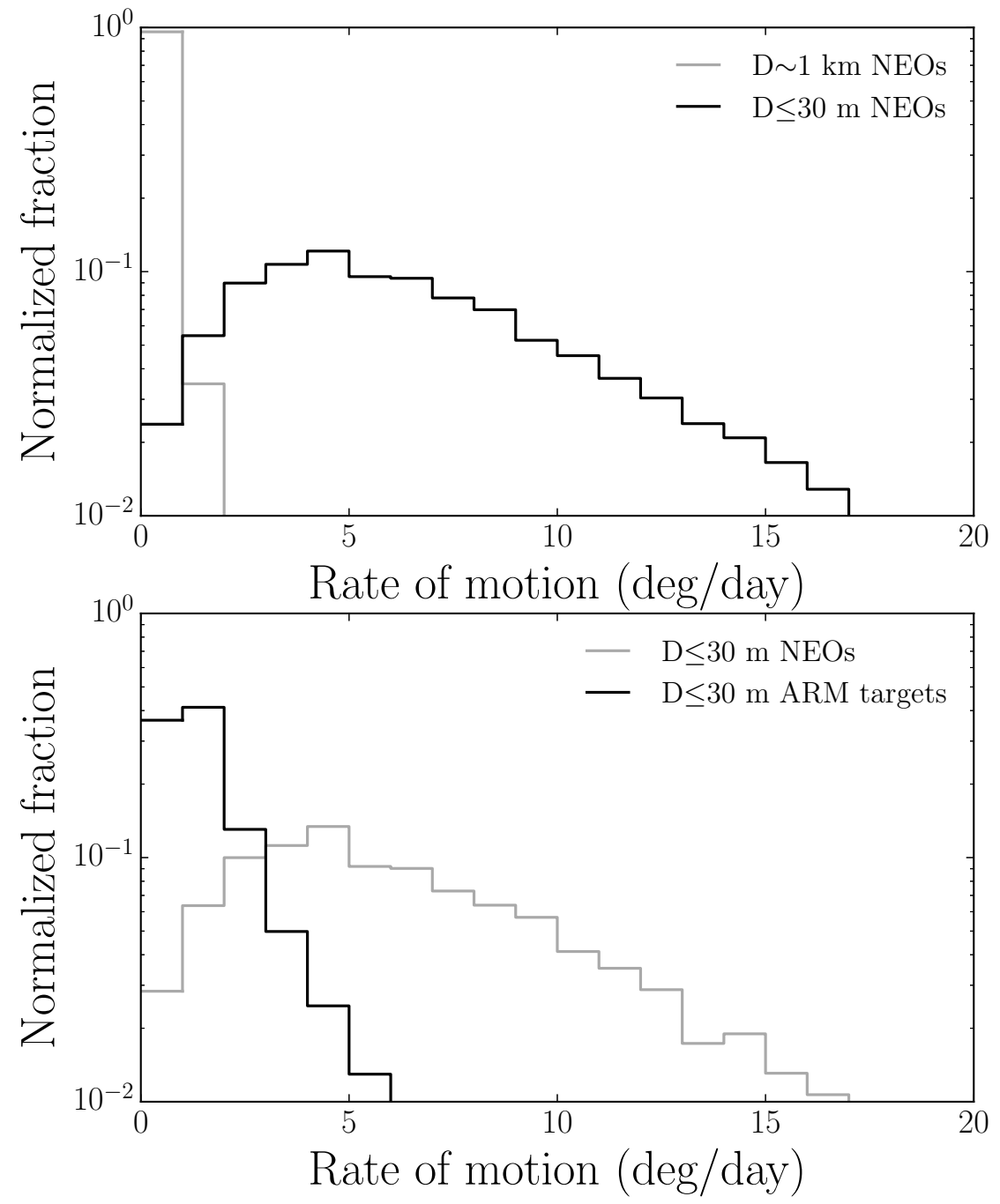

Fig. 5.- Rate of motion comparison between synthetic NEOs with $\sim 1 \mathrm{~km}$ and $\lesssim 30 \mathrm{~m}$ diameters ( corresponding to $\mathrm{H} \sim 17.5$ and $\mathrm{H}>27$ respectively, top panel) and synthetic NEOs and ARM targets with $\mathrm{D} \lesssim 30 \mathrm{~m}(\mathrm{H}>27)$ detected by PS1* (bottom panel). The largest ARM targets are significantly slower than NEOs at the same size. Data shown are before trailing loss implementation. 


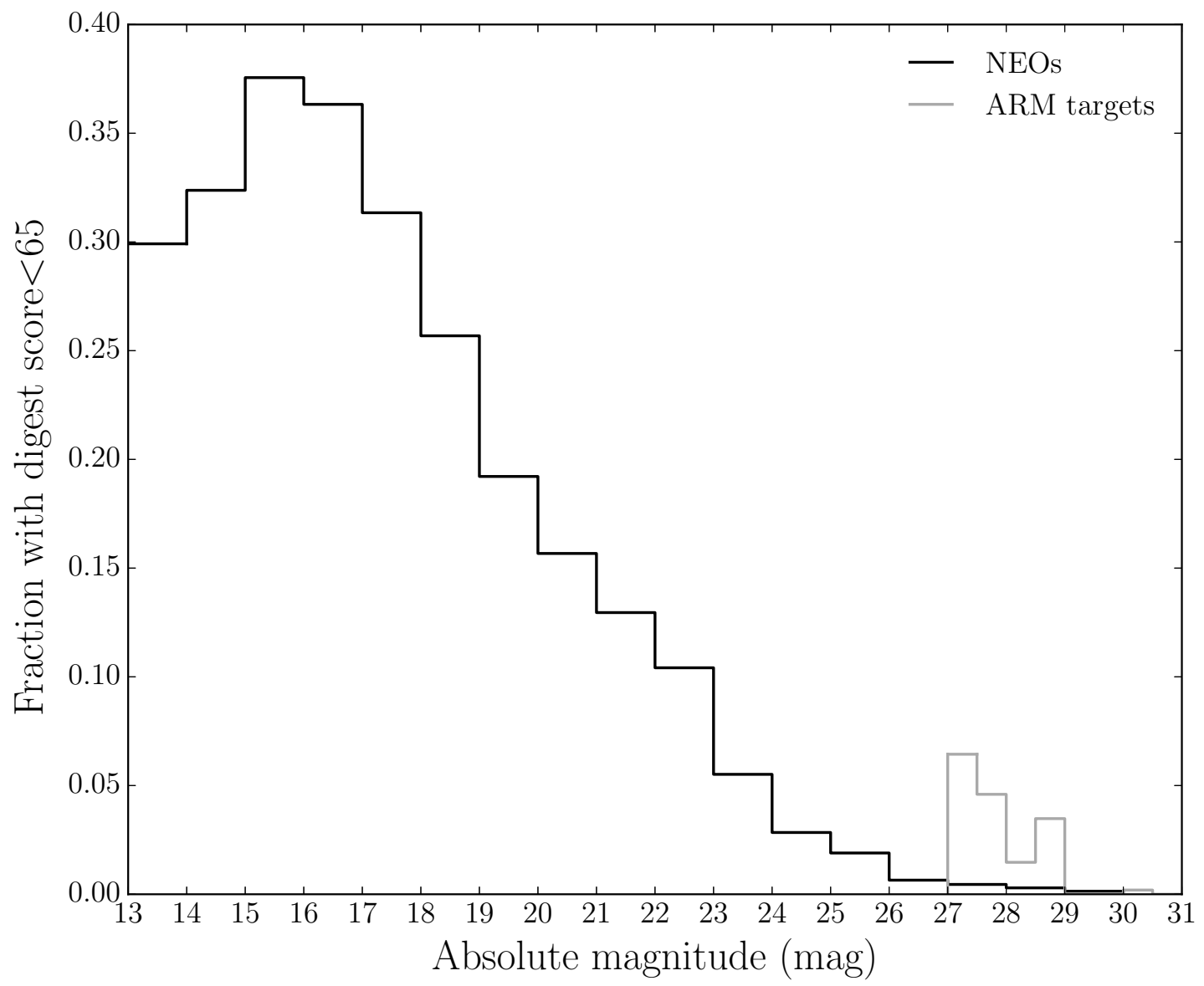

Fig. 6. - Fraction of NEOs and ARM targets with digest score $<65$ vs. absolute magnitude H. The fraction of NEOs detected with scores $<65$ which is the threshold of reporting them to MPC as NEOs is highest among the big bright objects that are detectable from greater distances and at lower apparent rate of motions than smaller objects. Almost 95\% of ARM targets have digest score higher then the MPC limit and are reported as NEOs. 

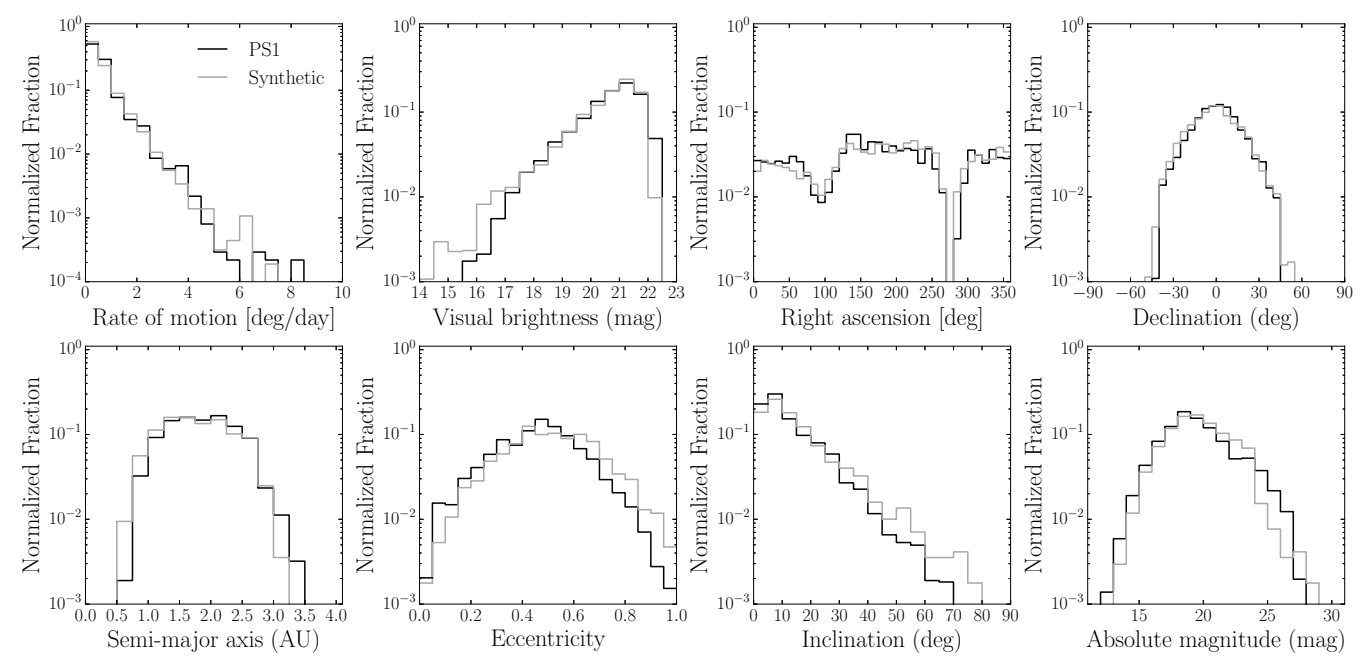

Fig. 7.- Comparison of rate of motion, visual magnitude, RA, DEC, a, e, i and absolute magnitude of synthetic NEOs from the PS1* simulation and the real Pan-STARRS1 data. 


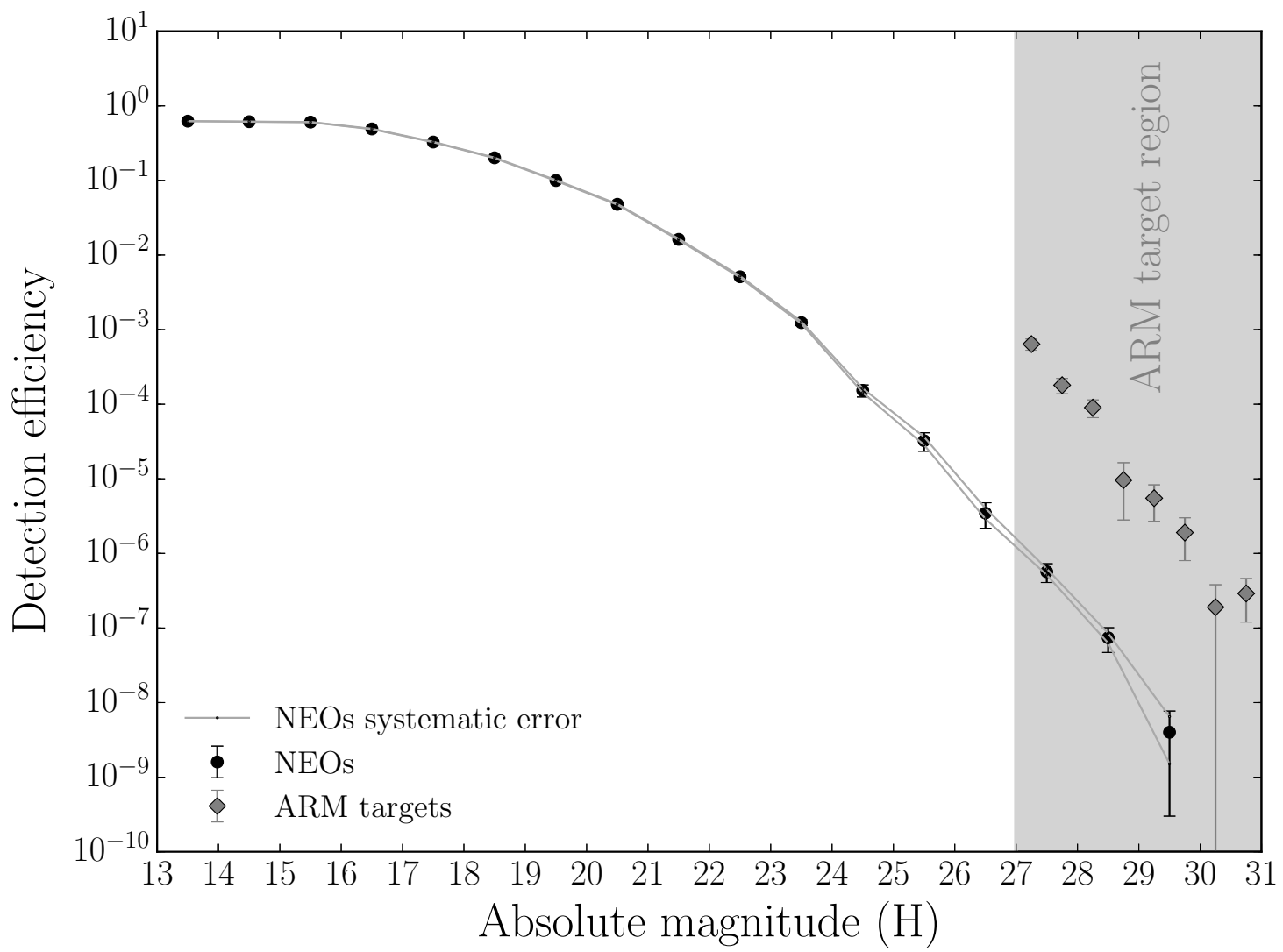

Fig. 8. - Absolute detection efficiency for NEO and ARM targets as a function of absolute magnitude for the PS1* simulation, i.e. the fraction of each population that would be detected during the survey duration (January 12014 - May 30 2015). 


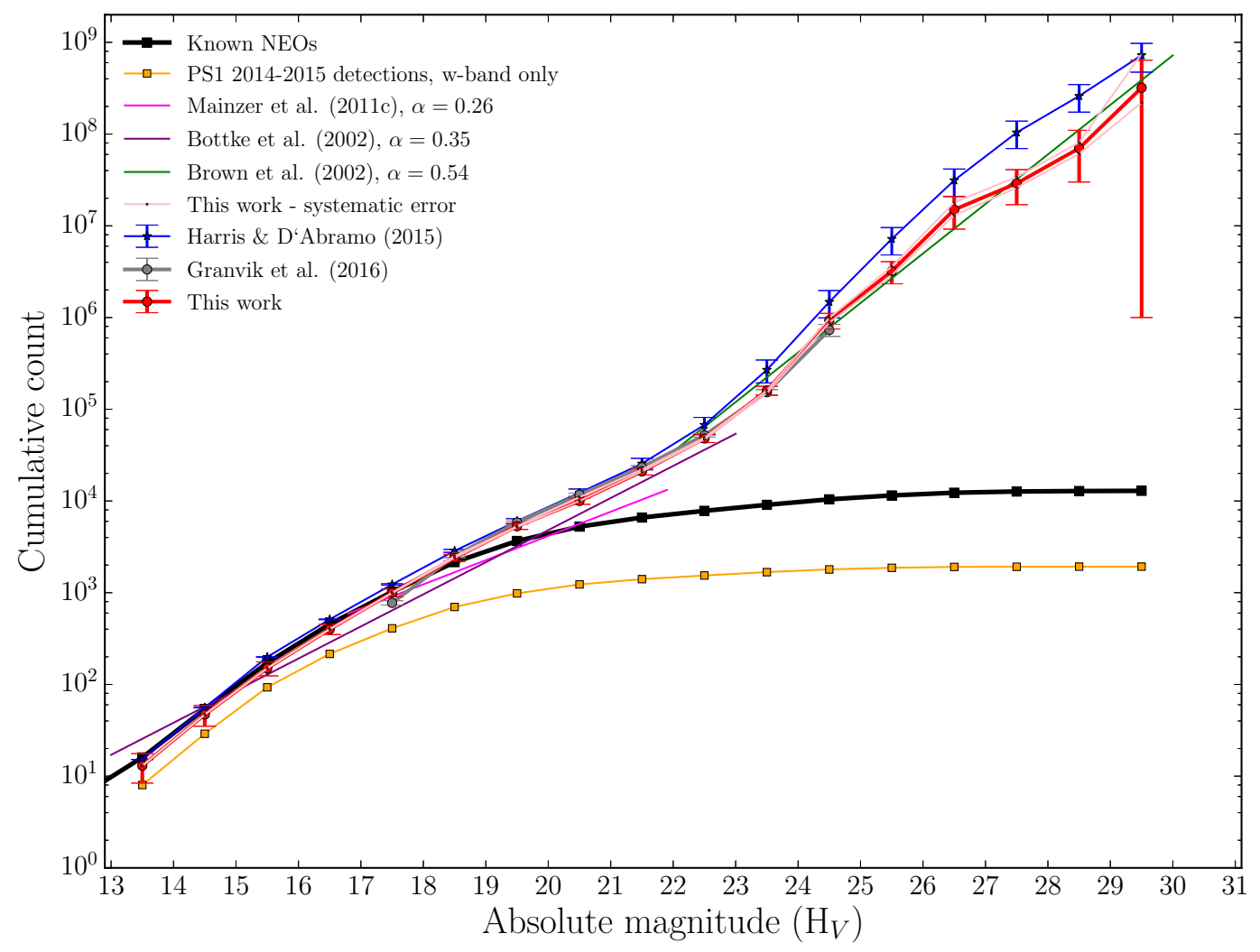

Fig. 9.- Our derived NEO SFD from Pan-STARRS1 data (§3.2) in comparison to other contemporary models and known NEOs. 


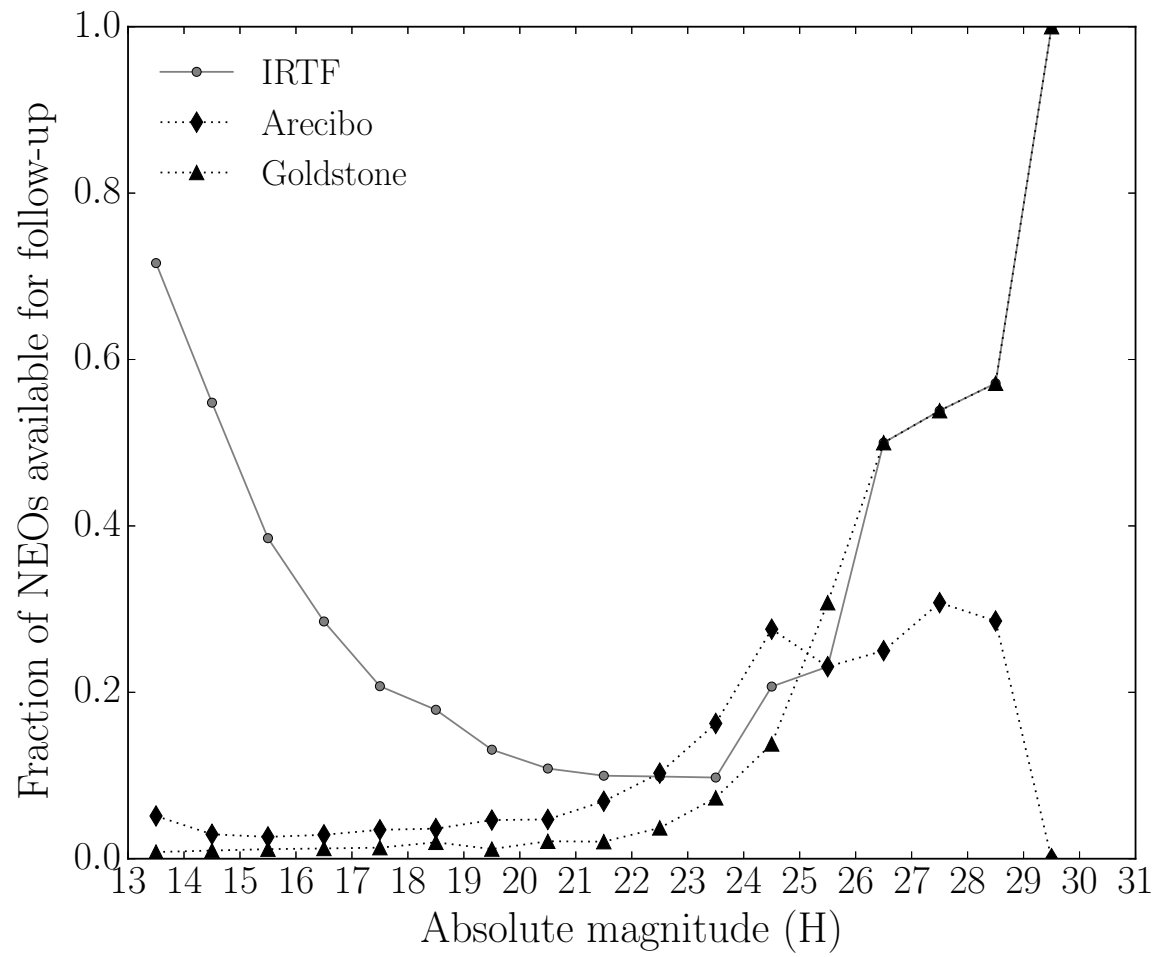

Fig. 10.- Fraction of NEOs accessible for follow-up observations with the IRTF telescope and the Arecibo and Goldstone radar facilities for 100 days after the discovery with PS1* . Virtually all NEOs are accessible to Pan-STARRS1 and CFHT after their discovery. 

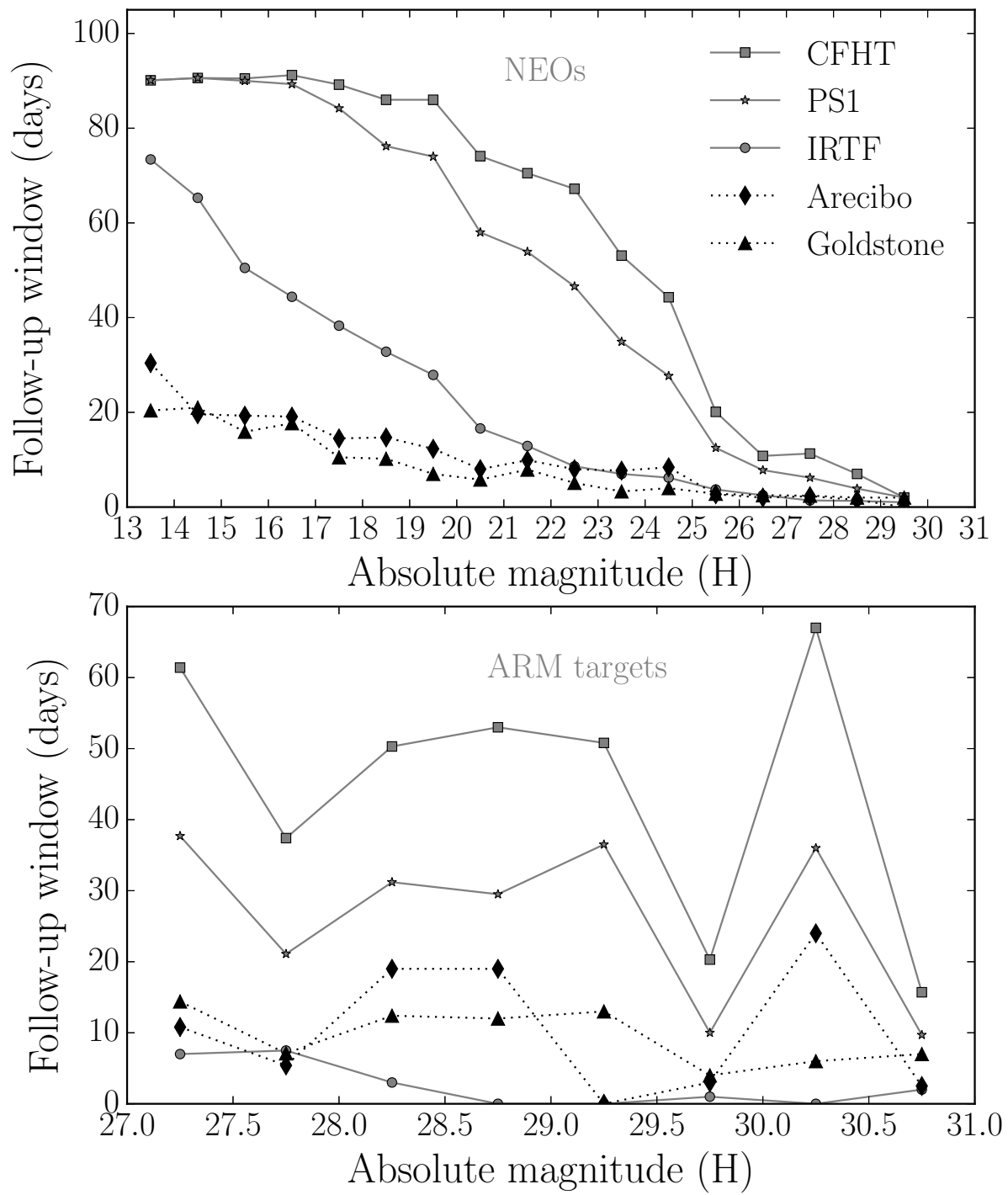

Fig. 11.- Average follow-up window duration for NEOs and ARM targets by the NASA IRTF telescope with SPEX, Pan-STARRS1 CFHT and Arecibo and Goldstone radars after discovery by Pan-STARRS1. 